\title{
Hamilton-Jacobi equations constrained on networks
}

\author{
Yves Achdou, Fabio Camilli, Alessandra Cutrì and \\ Nicoletta Tchou
}

\begin{abstract}
We consider continuous-state and continuous-time control problems where the admissible trajectories of the system are constrained to remain on a network. In our setting, the value function is continuous. We define a notion of constrained viscosity solution of Hamilton-Jacobi equations on the network and we study related comparison principles. Under suitable assumptions, we prove in particular that the value function is the unique constrained viscosity solution of the Hamilton-Jacobi equation on the network.
\end{abstract}

Mathematics Subject Classification (2010). Primary 35R02, 35F21, 35Q93; Secondary 34H05, 49J15.

Keywords. Optimal control, Graphs, Networks, Hamilton-Jacobi equations, Viscosity solutions.

\section{Introduction}

A network (or a graph) is a set of items, referred to as vertices or nodes, with connections between them referred to as edges. The main tools for the study of networks come from combinatorics and graph theory. But in the recent years there is an increasing interest in the investigation of dynamical systems and differential equation on networks, in particular in connection with problem of data transmission and traffic management (see for example Garavello and Piccoli [9], Engel et al. [6]). In this perspective, the study of control problems on networks has interesting applications in various fields. Note that partial differential operators on ramified spaces have also been investigated, see e.g. $[14,15]$.

A typical optimal control problem is the minimum time problem, which consists of finding the shortest path between an initial position and a given target set. If the running cost is a fixed constant for each edge and the dynamics can go from one vertex to an adjacent one at each time step, the corresponding discrete-state discrete-time control problem can be studied via graph theory 
and matrix analysis. If instead the cost changes in a continuous way along the edges and the dynamics is continuous in time, the minimum time problem can be seen as a continuous-state continuous-time control problem where the admissible trajectories of the system are constrained to remain on the network. While control problems with state constrained in closures of open sets are well studied $[4,11,17,18]$ there is to our knowledge much fewer literature on problems on networks: we very recently became aware of the thesis of Schieborn [16] devoted to the eikonal equation on networks, with an approach different from the one presented below. We also would like to mention the very recent preprint by Imbert, Monneau and Zidani [10] on an Hamilton-Jacobi approach to junction problems and traffic flows, in which the authors assume that the Hamiltonians associated with each edge do not depend on the state variable but may jump at the crosspoints. Their assumptions and their technique for proving the uniqueness of the viscosity solution greatly differs from ours, see our comment at the beginning of Sect. 5.3. The results of Frankowska and Plaskacz $[7,8]$ do apply to some closed sets with empty interior, but not to networks with crosspoints (except in very particular cases).

The aim of this paper is therefore to study optimal control problems whose dynamics is constrained to a network and the related Hamilton-JacobiBellman equation. Note that other types of optimal control problems could be considered as well, leading to other boundary conditions at the endpoints of the network. In most of the paper, we will consider for simplicity the model given by a star-shaped network, i.e. straight edges intersecting at the origin, see Fig. 1. This simple model problem already contains most of the difficulties that we have to face in more general situations. We shall briefly discuss more general networks with a finite number of vertices in Remark 5.5. Since the dynamics is constrained to the network, the velocities tangent to the network vary from one edge to another, hence the set of the admissible controls depends on the state of the system. If the set of admissible controls varies in a continuous way, the corresponding control problem can be studied via standard viscosity solution techniques (see Koike[12]). But for a network, the set of admissible controls drastically changes from a point in the interior of an edge, where only one direction is admissible (with possibly positive and negative velocities), to a vertex (or crosspoint) where the admissible directions are given by all the edges connected to it. Therefore, even if the data of the problem are regular, the corresponding Hamiltonian when restricted to the network has a discontinuous structure. Problem with discontinuous Hamiltonians have been recently studied by various authors (see e.g. Bressan and Hong [3], Deckelnick and Elliott [5], Koike [12], Soravia [19], Tourin [20]), but the approaches and the results considered in these papers do not seem to be applicable because of the particular structure of the considered domain.

Assuming that the set of the admissible control laws - i.e. the control laws for which the corresponding trajectory remains on the graph - is not empty, the control problem is well posed and the corresponding value function satisfies a dynamic programming principle. We introduce a first set of assumptions 
which guarantees that the value function is continuous on the network (with respect to the intrinsic geodetic distance).

The next step is to introduce a definition of weak solution which may ensure the uniqueness of the continuous solution via a comparison theorem. While in the interior of an edge we can test the equation with a smooth test function as in the standard case, the main difficulties arise at the vertices where the network does not allow a regular differential structure. At a vertex, we consider a concept of derivative similar to that of Dini's derivative, see for example [2], hence regular test functions are the ones which admit derivatives in the directions of the edges adjacent to the node. Using the previously mentioned class of test functions, we give a definition of viscosity solution on the network of the associated Hamilton-Jacobi equation, see (4.1). Note that at the crosspoints of the network, the definition of the Hamiltonian has to be particular, in order to take into account all the possible directions. It is worth to observe that this definition reduces to the classical one of viscosity solution if the graph is composed of two parallel segments entering in a node, see [2].

An important part of the present paper is devoted to proving comparison principles under fairly general assumptions: for example, our results apply to the case when on each edge of the network, the running cost is some power of the velocity, with exponents that may vary (within a suitable set) from one edge to another. In the proofs, the classical doubling technique of viscosity solution theory, see [13], is still used, but here, the choice of the penalty term in this argument requires a special care: we will see that the chosen penalty function is generally not symmetric w.r.t. the doubled variables, and that, to cope with the discontinuity in the Hamiltonians, it may depend on the small parameter $\varepsilon$ used in the method. In the simplest case, i.e. when the running cost does not depend on the control, the penalty term will be connected to the minimal time function with the dynamics frozen at the vertex; moreover, it will be bounded from above and below by some factor times the squared intrinsic geodetic distance, which, fixed one argument, is a regular test function of the other argument in the sense mentioned above.

We conclude observing that this paper is a first attempt to study Hamilton-Jacobi-Bellman equations and viscosity solutions on a network, and many questions remain to be studied, such as sub and super optimality principles, discontinuous viscosity solutions, stochastic control problems.

The paper is organized as follows: the control problem and the basic assumptions are set in Sect. 2. In Sect. 3, we define useful notions. In Sect. 4, we propose a definition of viscosity solutions of the Hamilton-Jacobi equation on the network, and we prove that the value function of the control problem is a viscosity solution. Comparison principles are studied in Sect. 5 .

\section{Setting of the problem and basic assumptions}

We consider a planar network with a finite number of edges and vertices. A network in $\mathbb{R}^{2}$ is a pair $(\mathcal{V}, \mathcal{E})$ where

(i) $\mathcal{V}$ is a finite subset of $\mathbb{R}^{2}$ whose elements are said vertices 
(ii) $\mathcal{E}$ is a finite set of regular arcs of $\mathbb{R}^{2}$, said edges, whose extrema are elements of $\mathcal{V}$.

We say that two vertices are adjacent if they are connected by an edge. We say that a vertex belongs to $\partial \mathcal{V}[$ resp., $\operatorname{int}(\mathcal{V})]$ if there is only one (resp., more than one) edge connected to it. We assume that the edges cross each other transversally. We denote by $\overline{\mathcal{G}}$ the union of all the edges in $\mathcal{E}$ and all the vertices in $\mathcal{V}$. We denote by $\mathcal{G}$ the set $\overline{\mathcal{G}} \backslash \partial \mathcal{V}$.

Except when explicitly mentioned, we focus for simplicity on the model case of a star-shaped network with $N$ straight edges, $N>1$, see Fig. 1, i.e.

$$
\mathcal{G}=\{O\} \cup \bigcup_{j=1}^{N} J_{j} \subset \mathbb{R}^{2}, \quad O=(0,0), \quad J_{j}=(0,1) e_{j},
$$

where $\left(e_{j}\right)_{j=1, \ldots, N}$ is a set of unit vectors in $\mathbb{R}^{2}$ s.t. $e_{j} \neq e_{k}$ if $j \neq k$. Note that $e_{j}=-e_{k}$ is possible. Then, $\partial \mathcal{V}=\left\{e_{j}, j=1, \ldots, N\right\}$ and $\operatorname{int}(\mathcal{V})=\{O\}$. We will use the notation $\partial \mathcal{G} \equiv \partial \mathcal{V}$. To avoid the trivial case of an interval, we assume that there is at least a pair $(j, k), j \neq k$ s.t. $e_{j}$ is not aligned with $e_{k}$. The general case will be dealt with in a forthcoming paper, where we will also consider structures made of several manifolds of different dimensions crossing each other transversally.

Hereafter, the notation $\mathbb{R}_{+}$stands for the interval $[0,+\infty)$.

For any $x \in \overline{\mathcal{G}}$, we denote by $T_{x}(\mathcal{G}) \subset \mathbb{R}^{2}$ the set of the tangent directions to the network, i.e. $T_{x}(\mathcal{G})=\mathbb{R} e_{i}, \forall x \in J_{i} ; T_{e_{i}}(\mathcal{G})=\mathbb{R}_{-} e_{i}$ and $T_{O}(\mathcal{G})=\cup_{i=1}^{N} \mathbb{R}_{+} e_{i}$. We now introduce the optimal control problem on $\overline{\mathcal{G}}$. We start by making some assumptions on the structure of the problem.

Call $B$ the closed unit ball of $\mathbb{R}^{2}$ centered at $O$. Take for $A$ a compact set of

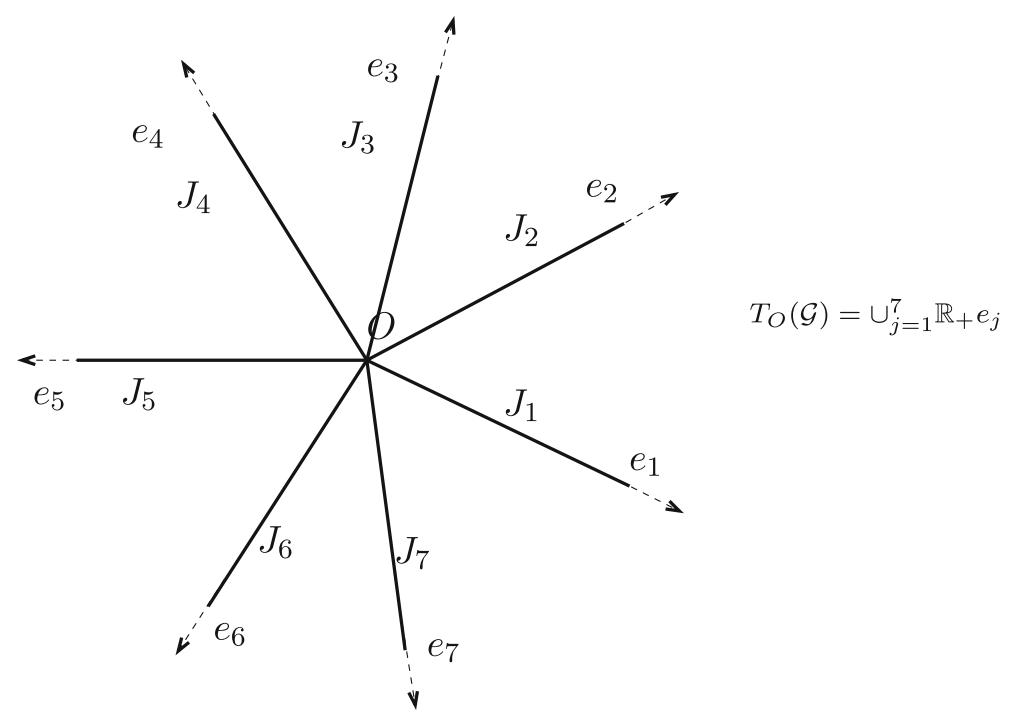

Figure 1. A star shaped network $\mathcal{G}$ 
$\mathbb{R}^{2}$ and a continuous function $f: B \times A \rightarrow \mathbb{R}^{2}$ such that

$$
|f(x, a)-f(y, a)| \leq L|x-y|, \quad \forall x, y \in B, a \in A .
$$

The continuity of $f$ implies that there exists $M>0$ such that

$$
|f(x, a)| \leq M, \quad \forall x \in B, a \in A .
$$

Denoting by $\mathcal{A}$ the class of the control laws, i.e. the set of measurable functions from $[0,+\infty)$ to $A$, we consider the dynamical system

$$
\left\{\begin{array}{l}
\dot{y}(t ; x, \alpha)=f(y(t ; x, \alpha), \alpha(t)), \quad t>0, \\
y(0)=x,
\end{array}\right.
$$

for $x \in \overline{\mathcal{G}}$ and $\alpha \in \mathcal{A}$. In order for (2.3) to make sense, additional assumptions will be made below.

Remark 2.1. We have chosen to parametrize the dynamics by a function $f$ defined on $B \times A$, i.e. on a much larger set than $\overline{\mathcal{G}} \times A$. We could also have defined $f$ on $\overline{\mathcal{G}} \times A$ only. This would have been equivalent since by Whitney extension theorem one can extend any Lipschitz function defined on $\overline{\mathcal{G}}$ to a Lipschitz function defined on $B$. In fact, all the assumptions made below on $f$ involve $\left.f\right|_{\overline{\mathcal{G}} \times A}$ only. Yet, it seemed to us that defining $f$ on $B \times A$ led to simpler notations.

We introduce the subset $\mathcal{A}_{x} \subset \mathcal{A}$ of the admissible control laws, i.e. the control laws for which the dynamics $(2.3)$ is constrained on the network $\overline{\mathcal{G}}$ :

$$
\mathcal{A}_{x}=\{\alpha \in \mathcal{A}: y(t ; x, \alpha) \in \overline{\mathcal{G}}, \quad \forall t>0\} .
$$

We also define for $x \in \overline{\mathcal{G}}$,

$$
A_{x}=\{a \in A \text { s.t. } \exists \theta>0: y(t ; x, a) \in \overline{\mathcal{G}}, \forall t, 0<t<\theta\} .
$$

From the continuity of $f$, we see that for all $a \in A_{x}, f(x, a) \in T_{x}(\mathcal{G})$.

Assumption 2.1. There exist non empty closed subsets $A^{j}$ of $A, j=1, \ldots, N$, such that

1.

$$
A=\bigcup_{j=1}^{N} A^{j}
$$

2. for $j \in\{1, \ldots, N\}, x \in J_{j}$ and $a \in A, f(x, a) \in \mathbb{R} e_{j}$ if and only if $a \in A^{j}$

3. for $j \in\{1, \ldots, N\}$,

$$
A_{e_{j}}=\left\{a \in A^{j}: f\left(e_{j}, a\right) \cdot e_{j} \leq 0\right\} \neq \emptyset, \text { and } \inf _{a \in A_{e_{j}}} f\left(e_{j}, a\right) \cdot e_{j}<0
$$

4 .

$$
A_{O}=\cup_{j=1}^{N}\left\{a \in A^{j}: f(O, a) \in \mathbb{R}_{+} e_{j}\right\} \neq \emptyset
$$

We easily obtain the following consequences of Assumption 2.1:

- for $j \in\{1, \ldots, N\}, x \in J_{j}$,

$$
A_{x}=A^{j}
$$


- for $j \in\{1, \ldots, N\}, x \in J_{j}$, there exists $\tau_{x}>0$ such that, for all $\alpha \in \mathcal{A}_{x}$,

$$
\alpha(t) \in A^{j} \text { for a.a. } t \in\left[0, \tau_{x}\right]
$$

- $\mathcal{A}_{x}$ is not empty for any $x \in \overline{\mathcal{G}}$. Hereafter, we will always consider $\alpha \in \mathcal{A}_{x}$ in (2.3).

Remark 2.2. Identity (2.6) says that the set of constant controls for which the trajectories leaving $x \in J_{j}$ stay in $\mathcal{G}$ for a positive time is nonempty and does not depend on $x \in J_{j}$. In (2.7), we see that for small durations, an admissible control law at $x \in \mathcal{G} \backslash\{O\}$ cannot take values outside $A_{x}$ (except maybe on a negligible set of times). The identity in (2.5) characterizes the set of constant controls for which the trajectories leaving $O$ stay in $\mathcal{G}$ for a positive time. Note that the identity in (2.5) is not a consequence of the previous points only when some edges are aligned. The assumption in (2.4) at the vertices in $\partial \mathcal{V}$ tells us that there exist controls which make the trajectory enter $\mathcal{G}$; this assumption is classical in the context of state constrained problems.

Assumption 2.2. There exist constants $\bar{\zeta}_{j}>0$ and $\underline{\zeta}_{j}>0, j=1, \ldots, N$, s.t.

$$
\overline{\mathrm{co}}\left(f\left(O, A^{j}\right)\right)=\left[-\underline{\zeta}_{j}, \bar{\zeta}_{j}\right] e_{j}, \quad \forall j=1, \ldots, N,
$$

where $\overline{\mathrm{co}}(F)$ stands for the closed convex hull of $F$.

Remark 2.3. Assumption 2.2 is on the controllability near $O$. We will see that it implies the continuity of the value function.

Remark 2.4. If $e_{j}=-e_{k}$ then, from (2.8) and the continuity of $f, \bar{\zeta}_{j}=\underline{\zeta}_{k}$ and $\bar{\zeta}_{k}=\underline{\zeta}_{j}$.

Example 2.1. Take $A=\cup_{j=1}^{N} \mathbb{R} e_{j} \cap B$ and $f(x, a)=g(x) a$ where $g: B \rightarrow \mathbb{R}$ is a positive and Lipschitz continuous function: we can see that all the assumptions above are satisfied. In particular, let us check that (2.7) holds in the present case: take $x \in \mathcal{G} \backslash\{O\}$, for example $x \in J_{1}$ and $\alpha \in \mathcal{A}_{x}$. With $M$ as in (2.2), take $\tau_{x}=|x| /(2 M)$. It is easy to see that $y(t ; x, \alpha) \in J_{1}$ for $t \in\left[0, \tau_{x}\right]$. This implies that $\int_{0}^{t} e_{1} \wedge f(y(s ; x, \alpha), \alpha(s)) d s=0$ for $t \in\left[0, \tau_{x}\right]$, and therefore $e_{1} \wedge f(y(t ; x, \alpha), \alpha(t))=g(y(t ; x, \alpha)) e_{1} \wedge \alpha(t)=0$ for almost all $t \in\left[0, \tau_{x}\right]$. Therefore, since $g$ is positive, $\alpha(t) \in A^{1}=A \cap \mathbb{R} e_{1}=A_{x}$ for almost all $t \in\left[0, \tau_{x}\right]$.

Example 2.2. Take $N$ unit vectors $\left(e_{j}\right)_{j=1, \ldots, N}$, with $e_{j}=\left(\cos \theta_{j}, \sin \theta_{j}\right), \theta_{j} \in$ $[0,2 \pi)$. Choose $\bar{\zeta}_{j}, \underline{\zeta}_{j} 2 N$ positive numbers such that $\bar{\zeta}_{j}=\underline{\zeta}_{k}$ and $\bar{\zeta}_{k}=\underline{\zeta}_{j}$ if $e_{j}=-e_{k}$. Take $A=\cup_{j=1}^{N} \mathbb{R} e_{j} \cap B$; let $\zeta: \mathbb{R} \rightarrow \mathbb{R}_{+}$be a $2 \pi$-periodic and continuous function such that $\zeta\left(\theta_{j}\right)=\bar{\zeta}_{j}$ and $\zeta\left(-\theta_{j}\right)=\underline{\zeta}_{j}, j=1, \ldots, N$; Choose $f(x, a)=g(x) \zeta(\theta) a$ where $a=|a|(\cos \theta, \sin \theta)$ and $g: B \rightarrow \mathbb{R}$ is a positive and Lipschitz continuous function. We can see that all the assumptions above are satisfied. 
Example 2.3. Choose $N$ unit vectors $\left(e_{j}\right)_{j=1, \ldots, N}$ and $2 N$ positive numbers $\bar{\zeta}_{j}, \underline{\zeta}_{j}$ as in Example 2.2. Take $A=\cup_{j=1}^{N} K e_{j}, K=\{-1,1\}$. Choose

$$
f(x, a)=g(x) \sum_{j=1}^{N}\left(-\underline{\zeta}_{j} 1_{a=-e_{j}}+\bar{\zeta}_{j} 1_{a=e_{j}}\right) e_{j}
$$

where $g: B \rightarrow \mathbb{R}$ is a positive and Lipschitz continuous function. We can see that all the assumptions above are satisfied.

Example 2.4. As a particular case of Example 2.3, one may take the cross shaped network $\mathcal{G}=\{O\} \cup \bigcup_{j=1}^{4} J_{j}, J_{1}=(0,1) e_{1}, J_{2}=-(0,1) e_{1}, J_{3}=$ $(0,1) e_{2}, J_{4}=-(0,1) e_{2}, e_{1}$ and $e_{2}$ being two orthogonal unit vectors. One may choose $A=K e_{1} \cup K e_{2}, K=\{-1,1\}$ and $f(x, a)=g(x) a$ where $g: B \rightarrow \mathbb{R}$ is a positive and Lipschitz continuous function.

Finally, we consider a continuous functions $\ell: B \times A \rightarrow \mathbb{R}$. We may suppose for simplicity that

$$
|\ell(x, a)| \leq M, \quad \forall x \in B, a \in A,
$$

where $M$ is the same constant as in (2.2). From the compactness of $B$ and $A$, there exists a modulus of continuity $\omega_{\ell}$ such that

$$
|\ell(x, a)-\ell(y, a)| \leq \omega_{\ell}(|x-y|), \quad \forall x, y \in B, a \in A .
$$

For $\lambda>0$, we consider the cost functional

$$
J(x, \alpha)=\int_{0}^{\infty} \ell(y(t ; x, \alpha), \alpha(t)) e^{-\lambda t} d t .
$$

The value function of the constrained control problem on the network is

$$
v(x)=\inf _{\alpha \in \mathcal{A}_{x}} J(x, \alpha), \quad x \in \overline{\mathcal{G}} .
$$

Assumption 2.1 and the assumptions on $\ell$ are enough for the dynamic programming principle:

$$
v(x)=\inf _{\alpha \in \mathcal{A}_{x}}\left\{\int_{0}^{t} \ell(y(s ; x, \alpha), \alpha(s)) e^{-\lambda s} d s+e^{-\lambda t} v(y(t ; x, \alpha))\right\} .
$$

The proof is standard along the arguments in Propositions III.2.5 or IV.5.5 in $[2]$.

Proposition 2.1. Under the assumptions above, the value function is continuous on $\overline{\mathcal{G}}$.

Proof. The proof is rather standard. It is given in [1].

We now give an example in which the value function is discontinuous: let $\left(e_{1}, e_{2}\right)$ be an orthonormal basis of $\mathbb{R}^{2}, \mathcal{G}=(0,1) e_{1} \cup\{O\} \cup(0,1) e_{2}, A=$ $\left\{0, e_{1}, e_{2}\right\}, f(x, a)=a(1-2|x|)$. Take $\ell(x, a)=1$ if $x_{2}=0$ and $\ell(x, a)=1-|x|$ 
if $x_{1}=0$. Assumption 2.2 is not satisfied. It is easy to compute the value function $v$ at $x=\left(x_{1}, x_{2}\right)$ : we have

$$
\begin{aligned}
& v\left(x_{1}, 0\right)=\frac{1}{\lambda}, \quad 0<x_{1} \leq 1, \\
& v\left(0, x_{2}\right)=\frac{1}{2 \lambda}+\frac{1-2 x_{2}}{4+2 \lambda}, \quad 0 \leq x_{2}<\frac{1}{2}, \\
& v\left(0, x_{2}\right)=\frac{1-x_{2}}{\lambda}, \quad \frac{1}{2} \leq x_{2} \leq 1 .
\end{aligned}
$$

The value function is discontinuous at $O$.

Hereafter, we will make a further assumption, mainly in order to obtain comparison principles in Sect. 5 below. Let us use the notation

$$
m_{O}=\min _{a \in A} \ell(O, a) .
$$

Assumption 2.3. The function $\ell$ satisfies: for all $j=1, \ldots, N$,

$$
\left(0, m_{O}\right) \in \overline{\mathrm{co}}\left((f(O, a), \ell(O, a)): a \in A^{j}\right) .
$$

Note that from Assumption 2.2, $0 \in \overline{\mathrm{co}}\left(\left(f(O, a): a \in A^{j}\right)\right.$, for any $j=$ $1, \ldots, N$,

Example 2.5. From Assumption 2.2, Assumption 2.3 is always satisfied if $\ell(O, a)$ does not depend on $a$.

Example 2.6. In the examples 2.1-2.4, we can take $\ell(x, a)=q(x)|a|^{\nu}+p(x)$, where $\nu \geq 0$ and $q$ and $p$ are continuous functions defined on $\overline{\mathcal{G}}$ with $q(O) \geq 0$.

\section{Preliminary notions for weak solutions}

\subsection{Test functions}

We introduce the class of the admissible test functions for the differential equation on the network

Definition 3.1. We say that a function $\varphi: \overline{\mathcal{G}} \rightarrow \mathbb{R}$ is an admissible test function and we write $\varphi \in \mathcal{R}(\mathcal{G})$ if

- $\varphi$ is continuous in $\overline{\mathcal{G}}$ and $\mathcal{C}^{1}$ in $\overline{\mathcal{G}} \backslash\{O\}$

- for any $j, j=1, \ldots, N,\left.\varphi\right|_{\overline{J_{j}}} \in \mathcal{C}^{1}\left(\overline{J_{j}}\right)$.

Therefore, for any $\zeta \in \mathbb{R}^{2}$ such that there exists a continuous function $z$ : $[0,1] \rightarrow \mathcal{G}$ and a sequence $\left(t_{n}\right)_{n \in \mathbb{N}}, 0<t_{n} \leq 1$ with $t_{n} \rightarrow 0$ and

$$
\lim _{n \rightarrow \infty} \frac{z\left(t_{n}\right)}{t_{n}}=\zeta
$$

the $\operatorname{limit}_{n \rightarrow \infty} \frac{\varphi\left(z\left(t_{n}\right)\right)-\varphi(O)}{t_{n}}$ exists and does not depend on $z$ and $\left(t_{n}\right)_{n \in \mathbb{N}}$. We define

$$
D \varphi(O, \zeta)=\lim _{n \rightarrow \infty} \frac{\varphi\left(z\left(t_{n}\right)\right)-\varphi(O)}{t_{n}} .
$$

If $x \in \mathcal{G} \backslash\{O\}$ and $\zeta \in T_{x}(\mathcal{G})$, we agree to write $D \varphi(x, \zeta)=D \varphi(x) \cdot \zeta$. 
Property 3.1. For any $\rho>0, D \varphi(O, \rho \zeta)=\rho D \varphi(O, \zeta)$.

Indeed, denoting by $\tau_{n}=t_{n} / \rho, \lim _{n \rightarrow \infty} z\left(t_{n}\right) / \tau_{n}=\rho \zeta$. Hence,

$$
\rho D \varphi(O, \zeta)=\lim _{n \rightarrow \infty} \frac{\varphi\left(z\left(t_{n}\right)\right)-\varphi(O)}{\tau_{n}}=D \varphi(O, \rho \zeta) .
$$

As shown below, property 3.1 is not true if $\rho<0$.

If $\varphi \in \mathcal{C}^{1}\left(\mathbb{R}^{2}\right)$, then $\varphi_{\mid \mathcal{G}} \in \mathcal{R}(\mathcal{G})$ and $D \varphi(O, \zeta)=D \varphi(O) \cdot \zeta$ for any $\zeta \in$ $\mathbb{R}_{+} e_{j}, j=1, \ldots, N$. If $e_{j}=-e_{k}$ for some $j \neq k \in\{1, \ldots, N\}, D \varphi\left(O, e_{j}\right)=$ $-D \varphi\left(O,-e_{j}\right)$.

If $\varphi$ is continuous and $\left.\varphi\right|_{\overline{\mathcal{G}} \cap \mathbb{R} e_{j}}$ is $\mathcal{C}^{1}$ for $j=1, \ldots, N$, then $\varphi \in \mathcal{R}(\mathcal{G})$ but the converse may not be true if two edges are aligned: for example, if $e_{j}=-e_{k}$ for some $j \neq k \in\{1, \ldots, N\}$, the function $x \mapsto b|x|$ belongs to $\mathcal{R}(\mathcal{G})$ and $D \varphi\left(O, e_{j}\right)=D \varphi\left(O,-e_{j}\right)=b$.

Property 3.2. If $\varphi=g \circ \psi$ with $g \in \mathcal{C}^{1}$ and $\psi \in \mathcal{R}(\mathcal{G})$, then $\varphi \in \mathcal{R}(\mathcal{G})$ and

$$
D \varphi(O, \zeta)=g^{\prime}(\psi(O)) D \psi(O, \zeta) \text {. }
$$

\subsection{Relaxed vector fields}

Definition 3.2. For $x \in \overline{\mathcal{G}}$, we define the set $\mathrm{FL}(x)$ as follows:

$$
\begin{aligned}
\mathrm{FL}(x) & \equiv \overline{\mathrm{co}}\left((f(x, a), \ell(x, a)): a \in A_{x}\right), \quad \text { if } x \in \mathcal{G} \backslash\{O\}, \\
\mathrm{FL}(O) & \equiv \bigcup_{j=1}^{N}\left(\overline{\mathrm{co}}\left((f(O, a), \ell(O, a)): a \in A^{j}\right) \cap\left(\mathbb{R}^{+} e_{j} \times \mathbb{R}\right)\right), \\
\mathrm{FL}\left(e_{j}\right) & \equiv \overline{\mathrm{co}}\left(\left(f\left(e_{j}, a\right), \ell\left(e_{j}, a\right)\right): a \in A^{j}\right) \cap\left(\mathbb{R}^{-} e_{j} \times \mathbb{R}\right) .
\end{aligned}
$$

\section{Viscosity solutions}

Hereafter, unless explicitly mentioned, we make all the assumptions of Sect. 2.

\subsection{Definition of viscosity solutions}

We now introduce the definition of a constrained viscosity solution of

$$
\lambda u(x)+\sup _{(\zeta, \xi) \in \mathrm{FL}(x)}\{-D u(x, \zeta)-\xi\}=0,
$$

in $\overline{\mathcal{G}}$, where $\mathrm{FL}(x)$ is defined in $(3.2-3.4)$.

Definition 4.1. $\quad$ - A bounded and upper semi-continuous function $u: \overline{\mathcal{G}} \rightarrow$ $\mathbb{R}$ is a subsolution of (4.1) in $\mathcal{G}$ if for any $x \in \mathcal{G}$, any $\varphi \in \mathcal{R}(\mathcal{G})$ s.t. $u-\varphi$ has a local maximum point at $x$, then

$$
\lambda u(x)+\sup _{(\zeta, \xi) \in \mathrm{FL}(x)}\{-D \varphi(x, \zeta)-\xi\} \leq 0 ;
$$

- A bounded and lower semi-continuous function $u: \overline{\mathcal{G}} \rightarrow \mathbb{R}$ is a supersolution of $(4.1)$ if for any $x \in \overline{\mathcal{G}}$, any $\varphi \in \mathcal{R}(\mathcal{G})$ s.t. $u-\varphi$ has a local minimum point at $x$, then

$$
\lambda u(x)+\sup _{(\zeta, \xi) \in \mathrm{FL}(x)}\{-D \varphi(x, \zeta)-\xi\} \geq 0 ;
$$


- A continuous function $u: \overline{\mathcal{G}} \rightarrow \mathbb{R}$ is a constrained viscosity solution of (4.1) in $\overline{\mathcal{G}}$ if it is a viscosity subsolution of (4.1) in $\mathcal{G}$ and supersolution of $(4.1)$ in $\overline{\mathcal{G}}$.

Remark 4.1. At $x \in \mathcal{G} \backslash\{O\}$, the notion of sub, respectively super-solution in Definition 4.1 is equivalent to the standard definition of viscosity sub, respectively super-solution of the equation

$$
\lambda u(x)+\sup _{a \in A_{x}}\{-f(x, a) \cdot D u-\ell(x, a)\}=0 .
$$

This is true because any test function in $\mathcal{R}(\mathcal{G})$ is $\mathcal{C}^{1}$ in a neighborhood of $x$ and because $\max _{(\zeta, \xi) \in \mathrm{FL}(x)}\{-D \varphi(x) \cdot \zeta-\xi\}$ is equal to $\sup _{a \in A_{x}}\{-D \varphi(x) \cdot f(x, a)-$ $\ell(x, a)\}$. Similarly, at $x \in \partial \mathcal{V}$, the notion of supersolution in $\overline{\mathcal{G}}$ is equivalent to the standard definition.

\subsection{An observation on a different possible formulation}

Before stating the existence result, we would like first to observe that thanks to Assumption 2.3, the present definition of viscosity solution is equivalent to a more general one, which is well adapted for the proof of existence: for that, we first define some larger relaxed vector fields:

Definition 4.2. For $x \in \overline{\mathcal{G}}$, we introduce the sets

$$
\tilde{f}(x)=\left\{\begin{array}{l|l}
\eta \in T_{x}(\mathcal{G}): & \begin{array}{l}
\exists\left(\alpha_{n}\right)_{n \in \mathbb{N}}, \alpha_{n} \in \mathcal{A}_{x}, \exists\left(t_{n}\right)_{n \in \mathbb{N}}, t_{n} \rightarrow 0^{+} \\
\text {s.t. } \\
\lim _{n \rightarrow \infty} \frac{1}{t_{n}} \int_{0}^{t_{n}} f\left(y\left(t ; x, \alpha_{n}\right), \alpha_{n}(t)\right) d t=\eta
\end{array}
\end{array}\right\}
$$

and

$$
\tilde{f \ell}(x)=\left\{\begin{array}{l|l}
(\eta, \mu) \in T_{x}(\mathcal{G}) \times \mathbb{R}: & \begin{array}{l}
\exists\left(\alpha_{n}\right)_{n \in \mathbb{N}}, \alpha_{n} \in \mathcal{A}_{x}, \exists\left(t_{n}\right)_{n \in \mathbb{N}}, t_{n} \rightarrow 0^{+} \\
\text {s.t. } \\
\lim _{n \rightarrow \infty} \frac{1}{t_{n}} \int_{0}^{t_{n}} f\left(y\left(t ; x, \alpha_{n}\right), \alpha_{n}(t)\right) d t=\eta, \\
\lim _{n \rightarrow \infty} \frac{1}{t_{n}} \int_{0}^{t_{n}} \ell\left(y\left(t ; x, \alpha_{n}\right), \alpha_{n}(t)\right) d t=\mu
\end{array}
\end{array}\right\} .
$$

Proposition 4.1. (a) Under all the assumptions made in Sect. 2 except Assumption 2.3,

$$
\begin{aligned}
& \tilde{f \ell}(x)=\mathrm{FL}(x), \quad \text { if } x \in \mathcal{G} \backslash\{O\}, \\
& \tilde{f} \ell(O) \supset \operatorname{FL}(O), \\
& \tilde{f} \ell\left(e_{j}\right)=\operatorname{FL}\left(e_{j}\right) .
\end{aligned}
$$

(b) Under all the assumptions made in Sect. 2,

1. For all $\zeta \in \tilde{f}(O) \cap \mathbb{R}_{+} e_{j}$, there exists $\xi \in \mathbb{R}$ such that $(\zeta, \xi) \in \mathrm{FL}(O)$.

2. For all $\zeta \in \tilde{f}(O)$,

$$
\min \{\mu:(\zeta, \mu) \in \mathrm{FL}(O)\}=\min \{\mu:(\zeta, \mu) \in \widetilde{f} \ell(O)\} .
$$

Proof. For keeping the section brief, the proof is postponed to Appendix A. 
Remark 4.2. The conclusions in point (b) of Proposition 4.1 hold if we replace Assumption 2.3 with the following: for any $i=1, \ldots, N$, the sets $A^{i}$ are of the form $A^{i}=\left[-\underline{\zeta}_{i}, \bar{\zeta}_{i}\right] e_{i}$ with $\underline{\zeta}_{i}>0$ and $\bar{\zeta}_{i}>0, f(O, a)=a$ if $a \in A^{i}$, and $a \mapsto \ell(O, a)$ is convex on $A^{i}$.

Corollary 4.2. Under all the assumptions made in Sect. 2 and in view of Proposition 4.1 (in particular point $b$. for $x=O$ ), (4.2) is equivalent to

$$
\lambda u(x)+\sup _{(\zeta, \xi) \in \widetilde{f} \ell(x)}\{-D \varphi(x, \zeta)-\xi\} \leq 0,
$$

and (4.3) is equivalent to

$$
\lambda u(x)+\sup _{(\zeta, \xi) \in \tilde{f} \ell(x)}\{-D \varphi(x, \zeta)-\xi\} \geq 0 .
$$

The same is true if the assumptions in Remark 4.2 are satisfied.

Remark 4.3. A definition of viscosity solutions using (4.7) and (4.8) would be more general in the case when Assumption 2.3 is not satisfied. However, we were not able so far to prove a comparison result without Assumption 2.3; thus, in the present work, there is no real reason for using the more general (and more abstract) definition, except for the proof of existence below.

\subsection{Existence}

Theorem 4.3. Under all the assumptions made in Sect. 2, the value function $v$ defined in (2.11) is a constrained viscosity solution of (4.1) in $\overline{\mathcal{G}}$.

Proof. From Corollary 4.2, we are going to use the relaxed vector fields $\widetilde{f} \ell(x)$ instead of FL $(x)$, i.e. to use (4.7) instead of (4.2) and (4.8) instead of (4.3) in the proof. We recall that $v$ satisfies the dynamic programming principle (2.12). The value function $v$ is a subsolution: it is enough to check that $v$ is a subsolution at $x=O$. Let $\varphi \in \mathcal{R}(\mathcal{G})$ be such that $v-\varphi$ has a maximum point at $O$, i.e.

$$
v(O)-v(z) \geq \varphi(O)-\varphi(z) \quad \forall z \in B_{O}(r) \cap \mathcal{G} .
$$

For $(\zeta, \xi) \in \tilde{f} \ell(O)$, there exists $\alpha_{n} \in \mathcal{A}_{O}$ and $t_{n} \rightarrow 0^{+}$such that

$$
\begin{aligned}
& \zeta=\lim _{n \rightarrow \infty} \frac{y\left(t_{n} ; O, \alpha_{n}\right)}{t_{n}}=\lim _{n \rightarrow \infty} \frac{1}{t_{n}} \int_{0}^{t_{n}} f\left(y\left(t ; O, \alpha_{n}\right), \alpha_{n}(t)\right) d t \\
& \xi=\lim _{n \rightarrow \infty} \frac{1}{t_{n}} \int_{0}^{t_{n}} \ell\left(y\left(t ; O, \alpha_{n}\right), \alpha_{n}(t)\right) d t .
\end{aligned}
$$

Take $T>0$ such that $y(t)=y(t ; O, \alpha) \in B_{O}(r) \cap \mathcal{G}$ for any $t \leq T$ and all $\alpha \in \mathcal{A}_{O}$. From (2.12)

$$
\begin{aligned}
& \varphi(O)-\varphi\left(y\left(t ; O, \alpha_{n}\right)\right) \leq v(O)-v\left(y\left(t ; O, \alpha_{n}\right)\right) \\
& \quad \leq \int_{0}^{t} \ell\left(y\left(s ; O, \alpha_{n}\right), \alpha_{n}(s)\right) e^{-\lambda s} d s+v\left(y\left(t ; O, \alpha_{n}\right)\right)\left(e^{-\lambda t}-1\right) .
\end{aligned}
$$

By (3.1),

$$
-D \varphi(O, \zeta)=\lim _{n \rightarrow \infty} \frac{\varphi(O)-\varphi\left(t_{n} \zeta\right)}{t_{n}} .
$$


Since $t_{n} \zeta=y\left(t_{n} ; O, \alpha_{n}\right)+o\left(t_{n}\right)$ and $\varphi$ is Lipschitz continuous, we deduce that

$$
-D \varphi(O, \zeta)=\lim _{n \rightarrow \infty} \frac{\varphi(O)-\varphi\left(y\left(t_{n} ; O, \alpha_{n}\right)\right)}{t_{n}} .
$$

On the other hand,

$$
\lim _{n \rightarrow \infty} \frac{1}{t_{n}} \int_{0}^{t_{n}} \ell\left(y\left(s ; O, \alpha_{n}\right), \alpha_{n}(s)\right) e^{-\lambda s} d s=\xi .
$$

Therefore

$$
-D \varphi(O, \zeta)-\xi \leq \lim _{n \rightarrow \infty} \frac{1}{t_{n}}\left(v\left(y\left(t_{n} ; O, \alpha_{n}\right)\right)\left(e^{-\lambda t_{n}}-1\right)\right)=-\lambda v(O) .
$$

Since the latter holds for any $(\zeta, \xi) \in \widetilde{f} \ell(O)$, we conclude that $v$ is a subsolution at $x=O$.

The value function $v$ is a supersolution. Let $\varphi \in \mathcal{R}(\mathcal{G})$ be such that $v-\varphi$ has a minimum point at $O$, i.e.

$$
v(O)-v(z) \leq \varphi(O)-\varphi(z) \quad \forall z \in B_{O}(r) \cap \mathcal{G} .
$$

We can always assume that $\varphi(O)=v(O)$ and $v(z) \geq \varphi(z), \forall z \in B_{O}(r) \cap \mathcal{G}$. From (2.12), for $\varepsilon>0$ and $t>0$, there exists $\alpha \in \mathcal{A}_{O}$ (depending on $\varepsilon$ and $t$ ) such that

$$
\begin{aligned}
v(O)+t \varepsilon & \geq \int_{0}^{t} \ell(y(s ; O, \alpha), \alpha(s)) e^{-\lambda s} d s+e^{-\lambda t} v(y(t ; O, \alpha)) \\
& \geq \int_{0}^{t} \ell(y(s ; O, \alpha), \alpha(s)) d s+e^{-\lambda t} v(y(t ; O, \alpha))+o(t),
\end{aligned}
$$

from the boundedness of $\ell$.

For $t$ sufficiently small, we get

$$
\begin{aligned}
& \varphi(O)-\varphi(y(t ; O, \alpha))-\int_{0}^{t} \ell(y(s ; O, \alpha), \alpha(s)) d s+\left(1-e^{-\lambda t}\right) v(y(t ; O, \alpha)) \\
& \quad \geq-t \varepsilon+o(t) .
\end{aligned}
$$

There exist sequences $t_{n} \rightarrow 0$ and $\alpha_{n} \in \mathcal{A}_{O}, \zeta$ and $\xi$ such that $\zeta=$ $\lim _{n \rightarrow \infty} \frac{y\left(t_{n}, O, \alpha_{n}\right)}{t_{n}}$ and $\xi=\lim _{n \rightarrow \infty} \frac{1}{t_{n}} \int_{0}^{t_{n}} \ell\left(y\left(s ; O, \alpha_{n}\right), \alpha_{n}(s)\right) d s$. Hence $(\zeta, \xi) \in \widetilde{f} \ell(O) \subset T_{O}(\mathcal{G}) \times \mathbb{R}$.

We clearly have

$$
\begin{aligned}
-\varepsilon+o(1) \leq & \frac{\varphi(O)-\varphi\left(y\left(t_{n} ; O, \alpha_{n}\right)\right)}{t_{n}}-\frac{1}{t_{n}} \int_{0}^{t_{n}} \ell\left(y\left(s ; O, \alpha_{n}\right), \alpha_{n}(s)\right) d s \\
& +\frac{\left(1-e^{-\lambda t_{n}}\right)}{t_{n}} v\left(y\left(t_{n} ; O, \alpha_{n}\right)\right) .
\end{aligned}
$$

But, as above, $\lim _{n \rightarrow \infty} \frac{\varphi(O)-\varphi\left(y\left(t_{n} ; O, \alpha_{n}\right)\right)}{t_{n}}=-D \varphi(O, \zeta)$. Therefore,

$$
\lambda v(O)+\sup _{(\eta, \mu) \in \tilde{f} \ell(O)}\{-D \varphi(O, \eta)-\mu\} \geq \lambda v(O)-D \varphi(O, \zeta)-\xi \geq-\varepsilon .
$$


From the arbitrariness of $\varepsilon$, we get that

$$
\lambda v(O)+\sup _{(\eta, \mu) \in \widetilde{f} \ell(O)}\{-D \varphi(O, \eta)-\mu\} \geq 0 .
$$

We conclude that $v$ is a supersolution at $x=O$.

\section{Comparison principle}

\subsection{Strategy}

We define the geodetic distance on $\overline{\mathcal{G}}$ by

$$
d(x, y)= \begin{cases}|x-y| & \text { if } x, y \in \overline{J_{j}}, j=1, \ldots, N \\ |x|+|y| & \text { if } x \in \overline{J_{i}}, y \in \overline{J_{j}}, i \neq j .\end{cases}
$$

Below, we give comparison principles under suitable assumptions. In their proofs, the main idea is to replace the standard penalizing term in the classical doubling of variables technique, see [2, p. 292], i.e. $\frac{d^{2}(x, y)}{\varepsilon}$ where $d(x, y)$ is the geodetic distance between $x$ and $y$, by a term $\frac{\tilde{d}_{\varepsilon}^{2}(x, y)}{\varepsilon}$ where $\tilde{d}_{\varepsilon}$ is a suitable nonsymmetric function such that $x \mapsto \tilde{d}_{\varepsilon}(x, y)$ and $y \mapsto \tilde{d}_{\varepsilon}(x, y)$ are regular in the sense of Definition 3.1.

For the reader's convenience, we first deal with the simplest case when the running cost does not depend on $a$ : in this case, the choice of $\tilde{d}_{\varepsilon}(x, y)$ is simple (it does not depend on $\varepsilon$ ), so some of the main ideas appear more clearly. In the second part, we give a more general result, for which the choice of the test function is more delicate.

\subsection{The simplest case: the running cost does not depend on $a$}

Here we assume that the running cost does not depend on $a$, so Assumption 2.3 is automatically satisfied.

In this case, it is clear that for any $x \in \overline{\mathcal{G}}, \mathrm{FL}(x)=\mathrm{F}(x) \times\{\ell(x)\}$, where

$$
\begin{aligned}
\mathrm{F}(x) & \equiv \overline{\mathrm{co}}\left(f(x, a): a \in A_{x}\right), \quad \text { if } x \in \mathcal{G} \backslash\{O\}, \\
\mathrm{F}(O) & \equiv \bigcup_{j=1}^{N}\left(\overline{\mathrm{co}}\left(f(O, a): a \in A^{j}\right) \cap \mathbb{R}^{+} e_{j}\right)=\bigcup_{j=1}^{N}\left[0, \bar{\zeta}_{j}\right] e_{j}, \\
\mathrm{~F}\left(e_{j}\right) & \equiv \overline{\mathrm{co}}\left(f\left(e_{j}, a\right): a \in A^{j}\right) \cap \mathbb{R}^{-} e_{j}, \quad j=1, \ldots, N,
\end{aligned}
$$

where the last identity in (5.2) is a direct consequence of Assumption 2.2. It is also easy to check that $(4.2)$ is equivalent to

$$
\lambda u(x)+\sup _{\zeta \in \mathrm{F}(x)}\{-D \varphi(x, \zeta)\}-\ell(x) \leq 0,
$$

and that (4.3) is equivalent to

$$
\lambda u(x)+\sup _{\zeta \in \mathrm{F}(x)}\{-D \varphi(x, \zeta)\}-\ell(x) \geq 0 .
$$

For proving the comparison principle, the following function will be useful: 
Definition 5.1. Let the function $\widetilde{d}: \overline{\mathcal{G}} \times \overline{\mathcal{G}} \rightarrow \mathbb{R}_{+}$be defined by

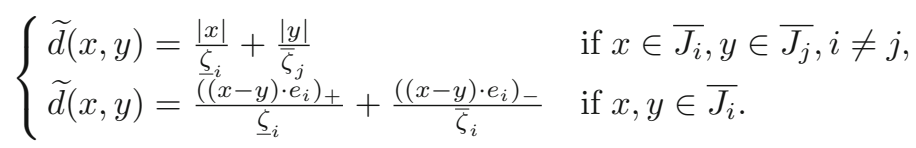

where $\underline{\zeta}_{i}$ and $\bar{\zeta}_{i}$ are the constants appearing in (2.8).

The following lemma can be easily checked:

Lemma 5.1. $\widetilde{d}$ is a Lipschitz continuous function on $\overline{\mathcal{G}} \times \overline{\mathcal{G}}$. For a fixed $x \in$ $\overline{\mathcal{G}}, y \mapsto \widetilde{d}^{2}(x, y)$ belongs to the class $\mathcal{R}(\mathcal{G})$ of test functions. Similarly, for a fixed $y \in \overline{\mathcal{G}}, x \mapsto \widetilde{d}^{2}(x, y)$ belongs to the class $\mathcal{R}(\mathcal{G})$ of test functions. There exist two positive constants $m$ and $M$ such that

$$
m d(x, y) \leq \widetilde{d}(x, y) \leq M d(x, y) .
$$

Remark 5.1. The quantity $\widetilde{d}(x, y)$ can be seen as the minimal time to reach $y$ from $x$, staying on $\mathcal{G}$, with velocities in $\cup_{j} \overline{\mathrm{co}}\left(f\left(O, A^{j}\right)\right)$. Note that, in general,

$$
\widetilde{d}(x, y) \neq \widetilde{d}(y, x) .
$$

Theorem 5.2. (Comparison principle) We assume that $\ell(x, a)$ does not depend on a. Under all the assumptions made in Sect. 2, if $u$ and $v$ are respectively $a$ subsolution of (4.1) in $\mathcal{G}$ and a supersolution of (4.1) in $\mathcal{G}$ such that

$$
u \leq v \quad \text { on } \partial \mathcal{G},
$$

then $u \leq v$ in $\overline{\mathcal{G}}$.

Proof. Note that $u-v$ is bounded and upper semi-continuous on $\overline{\mathcal{G}}$.

We assume by contradiction that there exist $x_{0} \in \overline{\mathcal{G}}, \chi>0$ such that

$$
u\left(x_{0}\right)-v\left(x_{0}\right)=\max _{\overline{\mathcal{G}}}(u-v)=\chi
$$

and we consider

$$
\Phi_{\varepsilon}(x, y)=u(x)-v(y)-\frac{\widetilde{d}^{2}(x, y)}{2 \varepsilon}, \quad x, y \in \overline{\mathcal{G}} .
$$

Let $\left(x_{\varepsilon}, y_{\varepsilon}\right)$ be a maximum point of $\Phi_{\varepsilon}$; we have

$$
\chi=\Phi_{\varepsilon}\left(x_{0}, x_{0}\right) \leq \Phi_{\varepsilon}\left(x_{\varepsilon}, y_{\varepsilon}\right) .
$$

From $\Phi_{\varepsilon}\left(x_{\varepsilon}, x_{\varepsilon}\right) \leq \Phi_{\varepsilon}\left(x_{\varepsilon}, y_{\varepsilon}\right)$, we get $\frac{\widetilde{d}^{2}\left(x_{\varepsilon}, y_{\varepsilon}\right)}{2 \varepsilon} \leq v\left(x_{\varepsilon}\right)-v\left(y_{\varepsilon}\right)$ and since $v$ is bounded,

$$
\widetilde{d}\left(x_{\varepsilon}, y_{\varepsilon}\right) \leq C \sqrt{\varepsilon}
$$

Hence $x_{\varepsilon}, y_{\varepsilon}$ converge for $\varepsilon \rightarrow 0$ to a point $\bar{x}$ and, by $(5.4), \bar{x} \in \mathcal{G}$. Therefore we can assume that for $\varepsilon$ sufficiently small, $x_{\varepsilon}, y_{\varepsilon} \in \mathcal{G}$ and, by standard arguments, we can prove that

$$
\lim _{\varepsilon \rightarrow 0} \frac{\widetilde{d}^{2}\left(x_{\varepsilon}, y_{\varepsilon}\right)}{2 \varepsilon}=0
$$


Moreover, $x \mapsto u(x)-\left(v\left(y_{\varepsilon}\right)+\frac{\widetilde{d}^{2}\left(x, y_{\varepsilon}\right)}{2 \varepsilon}\right)$ has a maximum point at $x_{\varepsilon}$ and by Lemma 5.1,

$$
\lambda u\left(x_{\varepsilon}\right)+\sup _{\zeta \in \mathrm{F}\left(x_{\varepsilon}\right)}\left\{-D\left(x \mapsto \frac{\widetilde{d}^{2}\left(x, y_{\varepsilon}\right)}{2 \varepsilon}\right)\left(x_{\varepsilon}, \zeta\right)\right\}-\ell\left(x_{\varepsilon}\right) \leq 0 .
$$

Similarly, $y \mapsto v(y)-\left(u\left(x_{\varepsilon}\right)-\frac{\widetilde{d}^{2}\left(x_{\varepsilon}, y\right)}{2 \varepsilon}\right)$ has a minimum at $y_{\varepsilon}$ and by Lemma 5.1,

$$
\lambda v\left(y_{\varepsilon}\right)+\sup _{\zeta \in \mathrm{F}\left(y_{\varepsilon}\right)}\left\{-D\left(y \mapsto-\frac{\widetilde{d}^{2}\left(x_{\varepsilon}, y\right)}{2 \varepsilon}\right)\left(y_{\varepsilon}, \zeta\right)\right\}-\ell\left(y_{\varepsilon}\right) \geq 0 .
$$

If $x_{\varepsilon}=y_{\varepsilon}$, subtracting (5.6) from (5.5) we get

$$
\lambda\left(u\left(x_{\varepsilon}\right)-v\left(x_{\varepsilon}\right)\right) \leq 0,
$$

and letting $\varepsilon \rightarrow 0$, we obtain the contradiction $\chi \leq 0$. Hence we can assume $x_{\varepsilon} \neq y_{\varepsilon}$.

1st case: $x_{\varepsilon} \neq O, y_{\varepsilon} \neq O$ : From (5.5) and (5.6), taking into account Remark 4.1, we get

$$
\begin{aligned}
\lambda\left(u\left(x_{\varepsilon}\right)-v\left(y_{\varepsilon}\right)\right) \leq & -\sup _{a \in A_{x_{\varepsilon}}}\left\{-D\left(x \mapsto \frac{\widetilde{d^{2}}\left(x, y_{\varepsilon}\right)}{2 \varepsilon}\right)\left(x_{\varepsilon}, f\left(x_{\varepsilon}, a\right)\right)\right\} \\
& +\sup _{a \in A_{y_{\varepsilon}}}\left\{-D\left(y \mapsto-\frac{\widetilde{d^{2}}\left(x_{\varepsilon}, y\right)}{2 \varepsilon}\right)\left(y_{\varepsilon}, f\left(y_{\varepsilon}, a\right)\right)\right\} \\
& +\ell\left(x_{\varepsilon}\right)-\ell\left(y_{\varepsilon}\right) .
\end{aligned}
$$

- If $x_{\varepsilon}, y_{\varepsilon}$ are on the same edge, for example, $x_{\varepsilon} \in \bar{J}_{1}$ and $y_{\varepsilon} \in \bar{J}_{1}$, the arguments are similar to those used in the classical theory of viscosity solutions; we give them for completeness. We make out two subcases:

- if $x_{\varepsilon} \cdot e_{1}>y_{\varepsilon} \cdot e_{1}$ then $\widetilde{d}^{2}\left(x_{\varepsilon}, y_{\varepsilon}\right)=\left|x_{\varepsilon}-y_{\varepsilon}\right|^{2} / \underline{\zeta}_{1}^{2}$, hence by (5.7), (2.1), (2.6) and (2.10),

$$
\begin{aligned}
& \lambda\left(u\left(x_{\varepsilon}\right)-v\left(y_{\varepsilon}\right)\right) \\
& \leq \frac{\widetilde{d}\left(x_{\varepsilon}, y_{\varepsilon}\right)}{\underline{\zeta}_{1}}\left(\sup _{a \in A_{y_{\varepsilon}}}\left\{-\frac{x_{\varepsilon}-y_{\varepsilon}}{\left|x_{\varepsilon}-y_{\varepsilon}\right|} \cdot f\left(y_{\varepsilon}, a\right)\right\}-\sup _{a \in A_{x_{\varepsilon}}}\left\{-\frac{x_{\varepsilon}-y_{\varepsilon}}{\left|x_{\varepsilon}-y_{\varepsilon}\right|} \cdot f\left(x_{\varepsilon}, a\right)\right\}\right) \\
& \quad+\ell\left(x_{\varepsilon}\right)-\ell\left(y_{\varepsilon}\right) \\
& \leq L \frac{\widetilde{d}^{2}\left(x_{\varepsilon}, y_{\varepsilon}\right)}{\varepsilon}+\omega_{\ell}\left(\left|x_{\varepsilon}-y_{\varepsilon}\right|\right),
\end{aligned}
$$

(note that $\left(x_{\varepsilon}-y_{\varepsilon}\right) /\left|x_{\varepsilon}-y_{\varepsilon}\right| \in T_{x_{\varepsilon}}(\mathcal{G})=T_{y_{\varepsilon}}(\mathcal{G})$ ), which yields the desired contradiction by having $\varepsilon$ tend to 0 .

- if $x_{\varepsilon} \cdot e_{1}<y_{\varepsilon} \cdot e_{1}$ then $\widetilde{d}^{2}\left(x_{\varepsilon}, y_{\varepsilon}\right)=\left|x_{\varepsilon}-y_{\varepsilon}\right|^{2} / \bar{\zeta}_{1}^{2}$, and we can repeat the argument immediately above. 
- If $x_{\varepsilon}, y_{\varepsilon}$ are not on the same edge, for example $x_{\varepsilon} \in \overline{J_{1}} \backslash\{O\}$ and $y_{\varepsilon} \in$ $\overline{J_{2}} \backslash\{O\}$ then $\widetilde{d}^{2}\left(x_{\varepsilon}, y_{\varepsilon}\right)=\left(\left|x_{\varepsilon}\right| / \underline{\zeta}_{1}+\left|y_{\varepsilon}\right| / \bar{\zeta}_{2}\right)^{2}$, hence by $(5.7)$

$$
\begin{aligned}
\lambda\left(u\left(x_{\varepsilon}\right)-v\left(y_{\varepsilon}\right)\right) \\
\quad \leq \frac{\widetilde{d}\left(x_{\varepsilon}, y_{\varepsilon}\right)}{\varepsilon}\left(\frac{1}{\bar{\zeta}_{2}} \sup _{a \in A_{y_{\varepsilon}}}\left\{\frac{y_{\varepsilon}}{\left|y_{\varepsilon}\right|} \cdot f\left(y_{\varepsilon}, a\right)\right\}-\frac{1}{\underline{\zeta}_{1}} \sup _{a \in A_{x_{\varepsilon}}}\left\{-\frac{x_{\varepsilon}}{\left|x_{\varepsilon}\right|} \cdot f\left(x_{\varepsilon}, a\right)\right\}\right) \\
\quad+\ell\left(x_{\varepsilon}\right)-\ell\left(y_{\varepsilon}\right),
\end{aligned}
$$

(note that $x_{\varepsilon} /\left|x_{\varepsilon}\right| \in T_{x_{\varepsilon}}(\mathcal{G})$ and $y_{\varepsilon} /\left|y_{\varepsilon}\right| \in T_{y_{\varepsilon}}(\mathcal{G})$ ). From (2.1), we get

$$
\begin{aligned}
& \lambda\left(u\left(x_{\varepsilon}\right)-v\left(y_{\varepsilon}\right)\right) \\
& \leq \frac{\tilde{d}\left(x_{\varepsilon}, y_{\varepsilon}\right)}{\varepsilon}\left(\frac{1}{\bar{\zeta}_{2}} \sup _{a \in A_{y_{\varepsilon}}}\left\{\frac{y_{\varepsilon}}{\left|y_{\varepsilon}\right|} \cdot f(O, a)\right\}-\frac{1}{\underline{\zeta}_{1}} \sup _{a \in A_{x_{\varepsilon}}}\left\{-\frac{x_{\varepsilon}}{\left|x_{\varepsilon}\right|} \cdot f(O, a)\right\}\right) \\
& \quad+\ell\left(x_{\varepsilon}\right)-\ell\left(y_{\varepsilon}\right)+L \frac{\widetilde{d}^{2}\left(x_{\varepsilon}, y_{\varepsilon}\right)}{\varepsilon} .
\end{aligned}
$$

From (2.6) and Assumption 2.2,

$$
-\frac{1}{\underline{\zeta}_{1}} \sup _{a \in A_{x_{\varepsilon}}}\left\{-\frac{x_{\varepsilon}}{\left|x_{\varepsilon}\right|} \cdot f(O, a)\right\}+\frac{1}{\bar{\zeta}_{2}} \sup _{a \in A_{y_{\varepsilon}}}\left\{\frac{y_{\varepsilon}}{\left|y_{\varepsilon}\right|} \cdot f(O, a)\right\}=-1+1=0
$$

and we obtain the desired contradiction from (5.8) and (2.10).

2nd case: $x_{\varepsilon}=O$ and $y_{\varepsilon} \neq O$ : Assume for example that $y_{\varepsilon} \in \overline{J_{2}} \backslash\{O\}$ (we proceed similarly in the other cases). Take $\zeta \in \mathrm{F}(O)$ where $\mathrm{F}(O)$ is given by (5.2). We know that $\overline{\mathrm{co}}\left(f(O, a): a \in A^{j}\right)$ is contained in $\mathbb{R} e_{j}$; therefore, $\delta(\zeta) \equiv D\left\{x \mapsto \widetilde{d}\left(x, y_{\varepsilon}\right)\right\}(O, \zeta)=-\frac{y_{\varepsilon}}{\bar{\zeta}_{2}\left|y_{\varepsilon}\right|} \cdot \zeta$ if $\zeta$ is aligned with $e_{2}$ and $\zeta \cdot e_{2}>0$ or $\delta(\zeta)=|\zeta| / \underline{\zeta}_{j}$ if $\zeta \in \mathrm{F}(O) \cap \mathbb{R} e_{j}$ is not aligned with $e_{2}$ or if $e_{j}$ is aligned with $e_{2}$ and $\zeta \cdot e_{2}<0$.

From (5.5) and (5.6), we get

$$
\begin{aligned}
\lambda\left(u(O)-v\left(y_{\varepsilon}\right)\right) \leq & \frac{\widetilde{d}\left(O, y_{\varepsilon}\right)}{\varepsilon}\left(-\sup _{\zeta \in \mathrm{F}(O)}\{-\delta(\zeta)\}+\sup _{a \in A_{y_{\varepsilon}}}\left\{\frac{y_{\varepsilon}}{\bar{\zeta}_{2}\left|y_{\varepsilon}\right|} \cdot f\left(y_{\varepsilon}, a\right)\right\}\right) \\
& +\ell(O)-\ell\left(y_{\varepsilon}\right) .
\end{aligned}
$$

From (2.1), we get that

$$
\begin{aligned}
\lambda\left(u(O)-v\left(y_{\varepsilon}\right)\right) \leq & \frac{\widetilde{d}\left(O, y_{\varepsilon}\right)}{\varepsilon}\left(-\sup _{\zeta \in \tilde{f}(O)}\{-\delta(\zeta)\}+\sup _{a \in A_{y_{\varepsilon}}}\left\{\frac{y_{\varepsilon}}{\bar{\zeta}_{2}\left|y_{\varepsilon}\right|} \cdot f(O, a)\right\}\right) \\
& +\ell(O)-\ell\left(y_{\varepsilon}\right)+L \frac{\widetilde{d^{2}}\left(O, y_{\varepsilon}\right)}{\varepsilon} .
\end{aligned}
$$


Thus, from (5.2), we get that

$$
\begin{aligned}
& -\sup _{\zeta \in \mathrm{F}(O)}\{-\delta(\zeta)\}+\sup _{a \in A_{y_{\varepsilon}}}\left\{\frac{y_{\varepsilon}}{\bar{\zeta}_{2}\left|y_{\varepsilon}\right|} \cdot f(O, a)\right\} \\
& =-\max _{j=1, \ldots, N} \max _{\zeta \in\left[0, \bar{\zeta}_{j}\right] e_{j}}(-\delta(\zeta))+\sup _{a \in A^{2}}\left\{\frac{e_{2} \cdot f(O, a)}{\bar{\zeta}_{2}}\right\} \\
& =-\max _{j=1, \ldots, N} \max _{\zeta \in\left[0, \bar{\zeta}_{j}\right] e_{j}}(-\delta(\zeta))+\bar{\zeta}_{2} / \bar{\zeta}_{2} \\
& =-1+1=0,
\end{aligned}
$$

where $\max _{j=1, \ldots, N} \max _{\zeta \in\left[0, \bar{\zeta}_{j}\right] e_{j}}(-\delta(\zeta))$ is obtained for $\zeta=\bar{\zeta}_{2} e_{2}$. This, with (2.10), yields the desired contradiction.

3rd case: $x_{\varepsilon} \neq O$ and $y_{\varepsilon}=O$ : Assume for example that $x_{\varepsilon} \in \overline{J_{2}} \backslash\{O\}$ (we proceed similarly in the other cases). Take $\zeta \in \mathrm{F}(O)$ where $\mathrm{F}(O)$ is given by (5.2). We know that $\delta(\zeta) \equiv D\left\{y \mapsto \widetilde{d}\left(x_{\varepsilon}, y\right)\right\}(O, \zeta)=-\frac{x_{\varepsilon}}{\underline{\zeta}_{2}\left|x_{\varepsilon}\right|} \cdot \zeta$ if $\zeta$ is aligned with $e_{2}$ and $\zeta \cdot e_{2}>0$ or $\delta(\zeta)=|\zeta| / \bar{\zeta}_{j}$ if $\zeta \in \mathrm{F}(O) \cap \mathbb{R} e_{j}$ is not aligned with $e_{2}$, or if $e_{j}$ is aligned with $e_{2}$ and $\zeta \cdot e_{2}<0$.

From (5.5) and (5.6), we get

$$
\begin{aligned}
& \lambda\left(u\left(x_{\varepsilon}\right)-v(O)\right) \\
& \quad \leq \frac{\widetilde{d}\left(x_{\varepsilon}, O\right)}{\varepsilon}\left(-\sup _{a \in A_{x_{\varepsilon}}}\left\{-\frac{x_{\varepsilon}}{\underline{\zeta}_{2}\left|x_{\varepsilon}\right|} \cdot f\left(x_{\varepsilon}, a\right)\right\}+\sup _{\zeta \in \mathrm{F}(O)}\{\delta(\zeta)\}\right) \\
& \quad+\ell\left(x_{\varepsilon}\right)-\ell(O) .
\end{aligned}
$$

This implies that

$$
\begin{aligned}
& \lambda\left(u\left(x_{\varepsilon}\right)-v(O)\right) \\
& \quad \leq \frac{\widetilde{d}\left(x_{\varepsilon}, O\right)}{\varepsilon}\left(-\sup _{a \in A_{x_{\varepsilon}}}\left\{-\frac{x_{\varepsilon}}{\underline{\zeta}_{2}\left|x_{\varepsilon}\right|} \cdot f(O, a)\right\}+\sup _{\zeta \in \mathrm{F}(O)}\{\delta(\zeta)\}\right) \\
& \quad+\ell\left(x_{\varepsilon}\right)-\ell(O)+L \frac{\widetilde{d}^{2}\left(x_{\varepsilon}, O\right)}{\varepsilon} .
\end{aligned}
$$

from (5.2), we get that

$$
\begin{aligned}
& \sup _{\zeta \in \mathrm{F}(O)}\{\delta(\zeta)\}-\sup _{a \in A_{x_{\varepsilon}}}\left\{-\frac{x_{\varepsilon}}{\underline{\zeta}_{2}\left|x_{\varepsilon}\right|} \cdot f(O, a)\right\} \\
& =\max _{j=1, \ldots, N} \max _{\zeta \in\left[0, \bar{\zeta}_{j}\right] e_{j}} \delta(\zeta)-\sup _{a \in A^{2}}\left\{\frac{-e_{2} \cdot f(O, a)}{\underline{\zeta}_{2}}\right\} \\
& =\max _{j \neq 2} \frac{\bar{\zeta}_{j}}{\bar{\zeta}_{j}}-1=0
\end{aligned}
$$

which, with (2.10), yields the desired contradiction. 


\subsection{More general cases}

Assumption 2.3 will enable us to use the argument of doubling the variables. This is in contrast with [10], where the authors do not make this assumption and have to rely on a special technique for proving the uniqueness of the viscosity solution.

Since the difficulties for proving the comparison results come from the junction point $O$, it will be helpful to freeze the state variables at $O$ and define the local Hamiltonians associated with the edges $J_{j}$ :

Definition 5.2. For $j=1, \ldots, N$, let $H_{j}: \mathbb{R} \mapsto \mathbb{R}$ and $H_{j}^{+}: \mathbb{R} \mapsto \mathbb{R}$ be the Hamiltonians:

$$
H_{j}(p)=\sup _{a \in A^{j}}\left(-p e_{j} \cdot f(O, a)-\ell(O, a)\right)
$$

and

$$
H_{j}^{+}(p)=\sup _{(\zeta, \xi) \in \overline{\operatorname{co}}\left\{(f(O, a), \ell(O, a)), a \in A^{j}\right\}, \zeta \cdot e_{j} \geq 0}\left(-p \zeta \cdot e_{j}-\xi\right)
$$

Lemma 5.3. Under Assumption 2.3,

$$
H_{j}(0)=-m_{O}, \quad \forall j=1, \ldots, N,
$$

where $m_{O}$ is defined in (2.13) and

$$
H_{j}(p)=H_{j}^{+}(p), \quad \forall p \leq 0 .
$$

Proof. It is clear that Assumption 2.3 implies (5.11). It also implies that for all $p \leq 0$ and $(\zeta, \xi) \in \overline{\mathrm{co}}\left\{(f(O, a), \ell(O, a)), a \in A^{j}\right\}$ such that $\zeta \cdot e_{j} \leq 0$,

$$
-p e_{j} \cdot \zeta-\xi \leq-m_{O} \leq H_{j}^{+}(p)
$$

and thus (5.12).

5.3.1. Assumptions. In what follows, we will need to somehow compare the local Hamiltonians on different sides of the junction $O$. For that, we will make one among the two assumptions below:

Assumption 5.1. There exist $2 N$ positive constants $K_{j}, k_{j}, j=1, \ldots, N$ such that

$$
H_{j}\left(-K_{j} p\right) \leq H_{i}\left(k_{i} p\right), \quad \forall p \in \mathbb{R}_{+}, \forall i, j, i \neq j .
$$

Assumption 5.2. For all $j=1, \ldots, N$, there exist real numbers $a_{j} \geq 0, b_{j}>$ $0, c_{j}>0, d_{j}>0, \zeta_{j}^{-}>0, \zeta_{j}^{+}>0, \alpha_{j}>1$ and $\gamma_{j}>1$ with

$$
2 \min _{i} \alpha_{i}>\max _{k} \gamma_{k} \text {, and } 2 \min _{k} \gamma_{k}>\max _{i} \alpha_{i} \text {, }
$$

such that

$$
H_{j}(-p)+m_{O} \leq \begin{cases}a_{j} p^{\alpha_{j}}, & 0 \leq p \leq \zeta_{j}^{-} \\ b_{j}\left(p-\zeta_{j}^{-}\right)+a_{j}\left(\zeta_{j}^{-}\right)^{\alpha_{j}}, & p \geq \zeta_{j}^{-}\end{cases}
$$

and

$$
H_{j}(p)+m_{O} \geq \begin{cases}c_{j} p^{\gamma_{j}}, & 0 \leq p \leq \zeta_{j}^{+} \\ d_{j}\left(p-\zeta_{j}^{+}\right)+c_{j}\left(\zeta_{j}^{+}\right)^{\gamma_{j}}, & p \geq \zeta_{j}^{+}\end{cases}
$$


Remark 5.2. For example, condition (5.14) holds if for all $i=1, \ldots, N, 1<$ $\alpha_{i} \leq 2$ and $1<\gamma_{i} \leq 2$.

Remark 5.3. In Assumption 5.2, we suppose in particular that for large values of $|q|$, the local Hamiltonians $H_{j}(q)$ are bounded from above or below by affine functions, depending on the sign of $q$, [see the second lines of (5.15) and (5.16)]. In fact, this part of the assumption is not mandatory for the comparison principle. Indeed, what follows holds if for $p \geq 0$,

$$
\begin{gathered}
H_{j}(-p)+m_{O} \leq a_{j} p^{\alpha_{j}}, \\
H_{j}(p)+m_{O} \geq c_{j} p^{\gamma_{j}} .
\end{gathered}
$$

However, Assumption 5.2 is coherent with the assumption made in Sect. 2 on the compactness of $A$.

We now give a series of examples, which show that in fairly general situations, at least one of the two assumptions above holds.

Example 5.1. Under the assumptions made in Sect. 2, Assumption 5.1 holds if $\ell(O, a)$ does not depend on $a$. Indeed, $H_{j}(p)=\underline{\zeta}_{j} p_{+}+\bar{\zeta}_{j} p_{-}-\ell(O)$, and we can choose $K_{j}=1 / \bar{\zeta}_{j}$ and $k_{j}=1 / \underline{\zeta}_{j}$. Therefore Assumption 5.1 covers the simplest case treated in the previous section.

Example 5.2. Take $N$ unit vectors $\left(e_{j}\right)_{j=1, \ldots, N}$. Only for simplicity, we assume that $e_{j}$ are pairwise linearly independent. Take $A=\cup_{j=1}^{N} \mathbb{R} e_{j} \cap B$. Take $2 N$ positive numbers $\underline{\zeta}_{j}$ and $\bar{\zeta}_{j}$ and a positive valued Lipschitz continuous function $\varphi$ defined in $B$ and bounded from below by a positive number. There exists a function $f: B \times A \rightarrow \mathbb{R}^{2}$ satisfying all the assumptions in Sect. 2, whose restriction to $A \cap \mathbb{R} e_{j}$ is

$$
\left.f\right|_{a \in \mathbb{R} e_{j}}(x, a)=\varphi(x)\left(1_{\left\{a \cdot e_{j} \leq 0\right\} \zeta_{j}}+1_{\left\{a \cdot e_{j} \geq 0\right\}} \bar{\zeta}_{j}\right) a .
$$

Let us assume that $\varphi(O)=1$. We take

$$
\ell(x, a)=q(x)|a|^{\nu}+m(x),
$$

where $\nu>1$ and $q$ and $m$ are continuous functions defined on $\overline{\mathcal{G}}$ with $q(O)>0$ : we have

$$
H_{j}(p)+m(O)= \begin{cases}-\bar{\zeta}_{j} p-q(O) & \text { if } \bar{\zeta}_{j} p \leq-\nu q(O), \\ (\nu-1) q(O)\left(\frac{|p| \bar{\zeta}_{j}}{\nu q(O)}\right)^{\frac{\nu}{\nu-1}} & \text { if }-\nu q(O) \leq \bar{\zeta}_{j} p \leq 0 \\ (\nu-1) q(O)\left(\frac{p \underline{\zeta}_{j}}{\nu q(O)}\right)^{\frac{\nu}{\nu-1}} & \text { if } 0 \leq \underline{\zeta}_{j} p \leq \nu q(O), \\ \underline{\zeta}_{j} p-q(O) & \text { if } \underline{\zeta}_{j} p \geq \nu q(O),\end{cases}
$$

then Assumption 5.1 is satisfied, with for example, $K_{j}=1 / \bar{\zeta}_{j}$ and $k_{j}=1 / \underline{\zeta}_{j}$.

Example 5.3. We keep everything as in Example 5.2, except that we suppose that the running cost is such that

$$
\left.\ell\right|_{x \in J_{j}, a \in \mathbb{R} e_{j}}(x, a)=m(x)+q_{j}(x)|a|^{\nu},
$$


where $q_{j}: J_{j} \rightarrow \mathbb{R}_{+}$are continuous functions such that $q_{j}(O)>0$ for all $j$. We have

$$
H_{j}(p)+m(O)= \begin{cases}-\bar{\zeta}_{j} p-q_{j}(O) & \text { if } \bar{\zeta}_{j} p \leq-\nu q_{j}(O), \\ (\nu-1) q_{j}(O)\left(\frac{|p| \bar{\zeta}_{j}}{\nu q_{j}(O)}\right)^{\frac{\nu}{\nu-1}} & \text { if }-\nu q_{j}(O) \leq \bar{\zeta}_{j} p \leq 0, \\ (\nu-1) q_{j}(O)\left(\frac{p \underline{\zeta}_{j}}{\nu q_{j}(O)}\right)^{\frac{\nu}{\nu-1}} & \text { if } 0 \leq \underline{\zeta}_{j} p \leq \nu q_{j}(O), \\ \underline{\zeta}_{j} p-q_{j}(O) & \text { if } \underline{\zeta}_{j} p \geq \nu q_{j}(O) .\end{cases}
$$

Let us choose the constants $k_{j}$ and $K_{j}$ such that, for all $i \neq j$,

$$
K_{j} \bar{\zeta}_{j}<k_{i} \underline{\zeta}_{i} \min \left(1, \frac{q_{j}(O)}{q_{i}(O)}\right) \text {. }
$$

Then, for $p \geq 0$, we can make out three cases:

case $1 . K_{j} p \geq \nu q_{j}(O) / \bar{\zeta}_{j}$. Thus, $k_{i} p \geq \nu q_{i}(O) / \underline{\zeta}_{i}$ and

$$
\begin{aligned}
H_{i}\left(k_{i} p\right)-H_{j}\left(-K_{j} p\right) & =\left(k_{i} \underline{\zeta}_{i}-K_{j} \bar{\zeta}_{j}\right) p+q_{j}(O)-q_{i}(O) \\
& \geq\left(k_{i} \underline{\zeta}_{i}-K_{j} \bar{\zeta}_{j}\right) \frac{q_{j}(O)}{K_{j} \bar{\zeta}_{j}}+q_{j}(O)-q_{i}(O) \\
& =q_{j}(O) \frac{k_{i} \underline{\zeta}_{i}}{K_{j} \bar{\zeta}_{j}}-q_{i}(O) \geq 0 .
\end{aligned}
$$

case 2. $k_{i} p \leq \nu q_{i}(O) / \underline{\zeta}_{i}$. Thus $K_{j} p \leq \nu q_{j}(O) / \bar{\zeta}_{j}$, and easy algebra shows that $H_{i}\left(k_{i} p\right)-H_{j}\left(-K_{j} p\right)$ has the same sign as

$\left(k_{i} \underline{\zeta}_{i} p\right)^{\nu} / q_{i}(O)-\left(K_{j} \bar{\zeta}_{j} p\right)^{\nu} / q_{j}(O)$, which is positive.

case 3. $\nu q_{i}(O) /\left(k_{i} \underline{\zeta}_{i}\right) \leq p \leq \nu q_{j}(O) /\left(K_{j} \bar{\zeta}_{j}\right)$. Comparing $H_{i}\left(k_{i} p\right)$

and $H_{j}\left(-K_{j} p\right)$ amounts to comparing respectively a linear function and a convex function: since $H_{i}\left(k_{i} p\right) \geq H_{j}\left(-K_{j} p\right)$ at the endpoints of the interval, the inequality is also true in the whole interval.

We have shown that Assumption 5.1 holds.

Example 5.4. We keep everything as in Example 5.3, except that the exponent $\nu$ may depend on $j$ :

$$
\left.\ell\right|_{x \in J_{j}, a \in \mathbb{R} e_{j}}(x, a)=m(x)+q_{j}(x)|a|^{\nu_{j}} .
$$

It can be checked that, if $q_{j}(O)>0$ and $\nu_{j} \in(0,1)$ for all $j$, then Assumptions 2.3 and 5.1 are both satisfied.

On the other hand, if $q_{j}(O)>0$ for all $j$ and if there exists a real number $s \geq 0$ such that $\nu_{j}^{*} \in\left(2^{s}, 2^{s+1}\right]$ for all $j$, where $\nu^{*}$ is the conjugate exponent of $\nu$, i.e. $1 / \nu+1 / \nu^{*}=1$, then Assumptions 2.3 and 5.2 are satisfied. This condition can be expressed as follows: there exists some nonnegative real number $s$ such that $\nu_{j} \in\left[1+\frac{1}{2^{s+1}-1}, 1+\frac{1}{2^{s}-1}\right.$ ), for all $j$ (in the particular case when $s=0$, this means that $\nu_{j} \in[2,+\infty)$, for all $j$ ). This example shows that, for Assumption 5.2 to hold, the power laws of the local Hamiltonian $H_{j}$ near $p=0$ may have different exponents, but that these exponents should not be too far from each other. 
5.3.2. The comparison principle. As explained in Sect. 5.1, we need to modify the geodetic distance in order to prove a comparison principle with the doubling of variables technique.

If Assumption 5.1 holds the situation is simple, because it is enough to take $\tilde{d}(x, y)$ defined as follows:

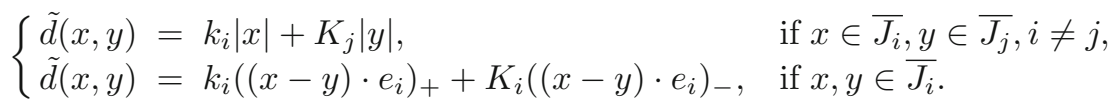

We see that function $\tilde{d}$ is similar to the one used in Sect. 5.2, and the proof of the comparison follows exactly the same lines.

Under Assumption 5.2, the situation is quite different, because the Hamiltonians $H_{j}(p)+m_{O}$ may have different behaviors when $p \rightarrow 0$, and we may have $\lim _{p \rightarrow 0+} \frac{H_{i}(p)+m_{O}}{H_{j}(-p)+m_{O}}=0$. To cope with this difficulty, we need to have the modified distance depend on $\varepsilon$. The following lemma plays an important role in the proof of the comparison principle under assumption 5.2.

Lemma 5.4. Let Assumptions 2.3 and 5.2 hold. Let $\eta_{j}$ and $\mu_{j}$ be respectively defined by

$$
\begin{aligned}
& \eta_{j}=\max \left(0, \frac{\alpha_{j}-\min _{1 \leq k \leq N} \gamma_{k}}{\alpha_{j}}\right), \\
& \mu_{j}=\max \left(1, \frac{\alpha_{j}}{2 \alpha_{j}-\max _{1 \leq k \leq N} \gamma_{k}}\right),
\end{aligned}
$$

there exists $k_{j}>0$ and $K_{j}>0$ such that, if $\tilde{d}_{\varepsilon}(x, y)$ is defined by

$$
\begin{cases}\tilde{d}_{\varepsilon}(x, y)=k_{i}|x|+K_{j} \varepsilon^{\eta_{j}}|y|^{\mu_{j}} & \text { if } x \in \overline{J_{i}}, y \in \overline{J_{j}}, i \neq j, \\ \tilde{d}_{\varepsilon}(x, y)=k_{i}\left((x-y) \cdot e_{i}\right)_{+}+K_{i} \varepsilon^{\eta_{i}}\left(\left((x-y) \cdot e_{i}\right)_{-}\right)^{\mu_{i}} & \text { if } x, y \in \overline{J_{i}},\end{cases}
$$

then, for all sequences $\left(x_{\varepsilon}, y_{\varepsilon}\right)$ such that $x_{\varepsilon} \in \overline{J_{j}}$ and $y_{\varepsilon} \in \overline{J_{i}}$ with $i \neq j$, and $\lim _{\varepsilon \rightarrow 0} \frac{\tilde{d}_{\varepsilon}^{2}\left(x_{\varepsilon}, y_{\varepsilon}\right)}{\varepsilon}=0$, it is possible to extract a subsequence such that

$$
H_{j}\left(D\left(x \mapsto \frac{\tilde{d}_{\varepsilon}^{2}\left(x, y_{\varepsilon}\right)}{2 \varepsilon}\right)\left(x_{\varepsilon}, e_{j}\right)\right) \geq H_{i}\left(D\left(y \mapsto-\frac{\tilde{d}_{\varepsilon}^{2}\left(x_{\varepsilon}, y\right)}{2 \varepsilon}\right)\left(y_{\varepsilon}, e_{i}\right)\right) .
$$

Proof. The proof mainly consists of studying real variable functions. For the reader's convenience, we give it in Appendix B.

Remark 5.4. In general, $\tilde{d}_{\varepsilon}(x, y) \neq \tilde{d}_{\varepsilon}(y, x)$.

\section{Preliminary observations}

1. We note that from (5.14), we have

$$
0 \leq \eta_{j}<\frac{1}{2}, \quad \forall j=1, \ldots, N .
$$


2. From (5.22), $\lim _{\varepsilon \rightarrow 0} \frac{\tilde{d}_{\varepsilon}^{2}\left(x_{\varepsilon}, y_{\varepsilon}\right)}{\varepsilon}=0$ implies that the geodetic distance between $x_{\varepsilon}$ and $y_{\varepsilon}$ tends to 0 when $\varepsilon \rightarrow 0$.

3. Moreover, $\max \left(\eta_{j}, \mu_{j}-1\right)=0$ if and only if $\alpha_{j}=\min _{k} \gamma_{k}$ and $\max _{k} \gamma_{k}=$ $\alpha_{j}$, i.e. $\gamma_{k}=\alpha_{j}$ for all $k=1, \ldots, N$.

The following lemma can be proved easily:

Lemma 5.5. The function $\tilde{d}_{\varepsilon}$ defined by (5.20) is a Lipschitz continuous function on $\overline{\mathcal{G}} \times \overline{\mathcal{G}}$. For a fixed $x \in \overline{\mathcal{G}}, y \mapsto \tilde{d}_{\varepsilon}^{2}(x, y)$ belongs to the class $\mathcal{R}(\mathcal{G})$ of test functions. Similarly, for a fixed $y \in \overline{\mathcal{G}}, x \mapsto \tilde{d}_{\varepsilon}^{2}(x, y)$ belongs to the class $\mathcal{R}(\mathcal{G})$ of test functions.

Theorem 5.6. (Comparison principle) Under all the assumptions made in Sect. 2 and either Assumption 5.1 or 5.2, if $u$ and $v$ are respectively a subsolution of (4.1) in $\mathcal{G}$ and a supersolution of (4.1) in $\mathcal{G}$ such that

$$
u \leq v \quad \text { on } \partial \mathcal{G}
$$

then $u \leq v$ in $\overline{\mathcal{G}}$.

Proof. When Assumption 5.1 holds, we have already said that the proof is exactly the same as that given in Sect. 5.2. Therefore, we focus on the case when Assumption 5.2 holds, in which a more involved argument is needed.

We assume by contradiction that there exist $x_{0} \in \overline{\mathcal{G}}, \chi>0$ such that $u\left(x_{0}\right)-$ $v\left(x_{0}\right)=\max _{\overline{\mathcal{G}}}(u-v)=\chi$, and we consider

$$
\Phi_{\varepsilon}(x, y)=u(x)-v(y)-\frac{\widetilde{d}_{\varepsilon}^{2}(x, y)}{2 \varepsilon}, \quad x, y \in \overline{\mathcal{G}},
$$

where $\widetilde{d}_{\varepsilon}$ is defined by $(5.20)$. Let $\left(x_{\varepsilon}, y_{\varepsilon}\right)$ be a maximum point of $\Phi_{\varepsilon}$; we have $\chi=\Phi_{\varepsilon}\left(x_{0}, x_{0}\right) \leq \Phi_{\varepsilon}\left(x_{\varepsilon}, y_{\varepsilon}\right)$. From $\Phi_{\varepsilon}\left(x_{\varepsilon}, x_{\varepsilon}\right) \leq \Phi_{\varepsilon}\left(x_{\varepsilon}, y_{\varepsilon}\right)$, we get $\frac{\widetilde{d}_{\varepsilon}^{2}\left(x_{\varepsilon}, y_{\varepsilon}\right)}{2 \varepsilon} \leq$ $v\left(x_{\varepsilon}\right)-v\left(y_{\varepsilon}\right)$ and since $v$ is bounded, $\widetilde{d}_{\varepsilon}\left(x_{\varepsilon}, y_{\varepsilon}\right) \leq C \sqrt{\varepsilon}$. Remember now that the exponents $\eta_{j}$ in the definition of $\widetilde{d}_{\varepsilon}$ belong to $[0,1 / 2)$, see $(5.22)$. Hence $x_{\varepsilon}, y_{\varepsilon}$ converge for $\varepsilon \rightarrow 0$ to a point $\bar{x}$ and, by $(5.23), \bar{x} \in \mathcal{G}$. Therefore we can assume that for $\varepsilon$ sufficiently small, $x_{\varepsilon}, y_{\varepsilon} \in \mathcal{G}$ and, by standard arguments, we can prove that $\lim _{\varepsilon \rightarrow 0} \frac{\widetilde{d}_{\varepsilon}^{2}\left(x_{\varepsilon}, y_{\varepsilon}\right)}{2 \varepsilon}=0$. Moreover, $x \mapsto u(x)-\left(v\left(y_{\varepsilon}\right)+\frac{\widetilde{d}_{\varepsilon}^{2}\left(x, y_{\varepsilon}\right)}{2 \varepsilon}\right)$ has a maximum point at $x_{\varepsilon}$ and by Lemma 5.5 ,

$$
\lambda u\left(x_{\varepsilon}\right)+\sup _{(\zeta, \xi) \in \mathrm{FL}\left(x_{\varepsilon}\right)}\left\{-D\left(x \mapsto \frac{\widetilde{d}_{\varepsilon}^{2}\left(x, y_{\varepsilon}\right)}{2 \varepsilon}\right)\left(x_{\varepsilon}, \zeta\right)-\xi\right\} \leq 0 .
$$

Similarly, $y \mapsto v(y)-\left(u\left(x_{\varepsilon}\right)-\frac{\widetilde{d}_{\varepsilon}^{2}\left(x_{\varepsilon}, y\right)}{2 \varepsilon}\right)$ has a minimum at $y_{\varepsilon}$ and by Lemma 5.5 ,

$$
\lambda v\left(y_{\varepsilon}\right)+\sup _{(\zeta, \xi) \in \mathrm{FL}\left(y_{\varepsilon}\right)}\left\{-D\left(y \mapsto-\frac{\widetilde{d}_{\varepsilon}^{2}\left(x_{\varepsilon}, y\right)}{2 \varepsilon}\right)\left(y_{\varepsilon}, \zeta\right)-\xi\right\} \geq 0 .
$$

If $x_{\varepsilon}=y_{\varepsilon}$, subtracting (5.25) from (5.24) we get

$$
\lambda\left(u\left(x_{\varepsilon}\right)-v\left(x_{\varepsilon}\right)\right) \leq 0
$$


and letting $\varepsilon \rightarrow 0$, we obtain the contradiction $\chi \leq 0$. Hence we can assume $x_{\varepsilon} \neq y_{\varepsilon}$.

Up to subsequence extraction, we distinguish the following possible cases:

1st case: $x_{\varepsilon} \neq O, y_{\varepsilon} \neq O$ : From (5.24) and (5.25), we get

$$
\begin{aligned}
& \lambda\left(u\left(x_{\varepsilon}\right)-v\left(y_{\varepsilon}\right)\right) \\
& \leq-\sup _{a \in A_{x_{\varepsilon}}}\left\{-D\left(x \mapsto \frac{\widetilde{d}_{\varepsilon}^{2}\left(x, y_{\varepsilon}\right)}{2 \varepsilon}\right)\left(x_{\varepsilon}, f\left(x_{\varepsilon}, a\right)\right)-\ell\left(x_{\varepsilon}, a\right)\right\} \\
& \quad+\sup _{a \in A_{y_{\varepsilon}}}\left\{-D\left(y \mapsto-\frac{\widetilde{d}_{\varepsilon}^{2}\left(x_{\varepsilon}, y\right)}{2 \varepsilon}\right)\left(y_{\varepsilon}, f\left(y_{\varepsilon}, a\right)\right)-\ell\left(y_{\varepsilon}, a\right)\right\} .
\end{aligned}
$$

- If $x_{\varepsilon}, y_{\varepsilon}$ are on the same edge, for example, $x_{\varepsilon} \in \bar{J}_{1}$ and $y_{\varepsilon} \in \bar{J}_{1}$, then we have two subcases

- if $x_{\varepsilon} \cdot e_{1}>y_{\varepsilon} \cdot e_{1}$, then $\widetilde{d}_{\varepsilon}\left(x_{\varepsilon}, y_{\varepsilon}\right)=k_{1}\left|x_{\varepsilon}-y_{\varepsilon}\right|$, hence by (5.26), (2.1), (2.6) and (2.10),

$$
\begin{aligned}
& \lambda\left(u\left(x_{\varepsilon}\right)-v\left(y_{\varepsilon}\right)\right) \\
& \leq-\sup _{a \in A_{x_{\varepsilon}}}\left\{-\frac{k_{1} \widetilde{d}_{\varepsilon}\left(x_{\varepsilon}, y_{\varepsilon}\right)}{\varepsilon} \frac{x_{\varepsilon}-y_{\varepsilon}}{\left|x_{\varepsilon}-y_{\varepsilon}\right|} \cdot f\left(x_{\varepsilon}, a\right)-\ell\left(x_{\varepsilon}, a\right)\right\} \\
& \quad+\sup _{a \in A_{y_{\varepsilon}}}\left\{-\frac{k_{1} \widetilde{d}_{\varepsilon}\left(x_{\varepsilon}, y_{\varepsilon}\right)}{\varepsilon} \frac{x_{\varepsilon}-y_{\varepsilon}}{\left|x_{\varepsilon}-y_{\varepsilon}\right|} \cdot f\left(y_{\varepsilon}, a\right)-\ell\left(y_{\varepsilon}, a\right)\right\} \\
& \leq L \frac{\widetilde{d}_{\varepsilon}^{2}\left(x_{\varepsilon}, y_{\varepsilon}\right)}{\varepsilon}+\omega_{\ell}\left(\left|x_{\varepsilon}-y_{\varepsilon}\right|\right),
\end{aligned}
$$

(note that $\left(x_{\varepsilon}-y_{\varepsilon}\right) /\left|x_{\varepsilon}-y_{\varepsilon}\right| \in T_{x_{\varepsilon}}(\mathcal{G})=T_{y_{\varepsilon}}(\mathcal{G})$ ), which yields the desired contradiction by having $\varepsilon$ tend to 0 .

- If $x_{\varepsilon} \cdot e_{1}<y_{\varepsilon} \cdot e_{1}$, then $\widetilde{d}_{\varepsilon}\left(x_{\varepsilon}, y_{\varepsilon}\right)=K_{1} \varepsilon^{\eta_{1}}\left|x_{\varepsilon}-y_{\varepsilon}\right|^{\mu_{1}}$ and

$$
\begin{aligned}
& \left|D\left(y \mapsto-\frac{\widetilde{d}_{\varepsilon}^{2}\left(x_{\varepsilon}, y\right)}{2 \varepsilon}\right)\left(y_{\varepsilon}, f\left(x_{\varepsilon}, a\right)-f\left(y_{\varepsilon}, a\right)\right)\right| \\
& \leq \frac{\widetilde{d}_{\varepsilon}\left(x_{\varepsilon}, y_{\varepsilon}\right)}{\varepsilon} K_{1} \mu_{1} \varepsilon^{\eta_{1}}\left|x_{\varepsilon}-y_{\varepsilon}\right|^{\mu_{1}-1}\left|f\left(x_{\varepsilon}, a\right)-f\left(y_{\varepsilon}, a\right)\right| \leq C \frac{\widetilde{d}_{\varepsilon}^{2}\left(x_{\varepsilon}, y_{\varepsilon}\right)}{\varepsilon}
\end{aligned}
$$

and we use the same argument as above.

- If $x_{\varepsilon}, y_{\varepsilon}$ are not on the same edge, for example $x_{\varepsilon} \in \overline{J_{1}} \backslash\{O\}$ and $y_{\varepsilon} \in$ $\overline{J_{2}} \backslash\{O\}$ then $\widetilde{d}_{\varepsilon}^{2}\left(x_{\varepsilon}, y_{\varepsilon}\right)=\left(k_{1}\left|x_{\varepsilon}\right|+K_{2} \varepsilon^{\eta_{2}}\left|y_{\varepsilon}\right|^{\mu_{2}}\right)^{2}$, hence by $(5.26)$

$$
\begin{aligned}
& \lambda\left(u\left(x_{\varepsilon}\right)-v\left(y_{\varepsilon}\right)\right) \\
& \leq-\sup _{a \in A_{x_{\varepsilon}}}\left\{-\frac{k_{1} \widetilde{d}_{\varepsilon}\left(x_{\varepsilon}, y_{\varepsilon}\right)}{\varepsilon} e_{1} \cdot f\left(x_{\varepsilon}, a\right)-\ell\left(x_{\varepsilon}, a\right)\right\} \\
& \quad+\sup _{a \in A_{y_{\varepsilon}}}\left\{\frac{K_{2} \mu_{2} \varepsilon^{\eta_{2}} \widetilde{d}_{\varepsilon}\left(x_{\varepsilon}, y_{\varepsilon}\right)}{\varepsilon}\left|y_{\varepsilon}\right|^{\mu_{2}-1} e_{2} \cdot f\left(y_{\varepsilon}, a\right)-\ell\left(y_{\varepsilon}, a\right)\right\}
\end{aligned}
$$


From (2.1), we get

$$
\begin{aligned}
\lambda\left(u\left(x_{\varepsilon}\right)-v\left(y_{\varepsilon}\right)\right) & \\
\leq & -H_{1}\left(\frac{k_{1} \widetilde{d}_{\varepsilon}\left(x_{\varepsilon}, y_{\varepsilon}\right)}{\varepsilon}\right)+H_{2}\left(-\frac{K_{2} \mu_{2} \varepsilon^{\eta_{2}} \widetilde{d}_{\varepsilon}\left(x_{\varepsilon}, y_{\varepsilon}\right)\left|y_{\varepsilon}\right|^{\mu_{2}-1}}{\varepsilon}\right) \\
& +C \frac{\widetilde{d}_{\varepsilon}^{2}\left(x_{\varepsilon}, y_{\varepsilon}\right)}{\varepsilon}+\omega_{\ell}\left(\left|x_{\varepsilon}\right|\right)+\omega_{\ell}\left(\left|y_{\varepsilon}\right|\right) \\
= & H_{2}\left(D\left(y \mapsto-\frac{\widetilde{d}_{\varepsilon}^{2}\left(x_{\varepsilon}, y\right)}{2 \varepsilon}\right)\left(y_{\varepsilon}, e_{2}\right)\right)-H_{1}\left(D\left(x \mapsto \frac{\widetilde{d}_{\varepsilon}^{2}\left(x, y_{\varepsilon}\right)}{2 \varepsilon}\right)\left(x_{\varepsilon}, e_{1}\right)\right) \\
& +C \frac{\widetilde{d_{\varepsilon}^{2}}\left(x_{\varepsilon}, y_{\varepsilon}\right)}{\varepsilon}+2 \omega_{\ell}\left(d\left(x_{\varepsilon}, y_{\varepsilon}\right)\right),
\end{aligned}
$$

and we obtain the desired contradiction from (5.21).

2nd case: $x_{\varepsilon}=O$ and $y_{\varepsilon} \neq O$ : Assume for example that $y_{\varepsilon} \in \overline{J_{2}} \backslash\{O\}$ (we proceed similarly in the other cases).

From (5.24) and (5.25), we get

$$
\begin{aligned}
& \lambda\left(u(O)-v\left(y_{\varepsilon}\right)\right) \\
& \leq-\sup _{(\zeta, \xi) \in \mathrm{FL}(O)}\left\{D\left(x \mapsto-\frac{\widetilde{d}_{\varepsilon}^{2}\left(x, y_{\varepsilon}\right)}{2 \varepsilon}\right)(O, \zeta)-\xi\right\} \\
& \quad+H_{2}\left(-\frac{K_{2} \mu_{2} \varepsilon^{\eta_{2}} \widetilde{d}_{\varepsilon}\left(O, y_{\varepsilon}\right)\left|y_{\varepsilon}\right|^{\mu_{2}-1}}{\varepsilon}\right)+C \frac{\widetilde{d}_{\varepsilon}^{2}\left(O, y_{\varepsilon}\right)}{\varepsilon}+\omega_{\ell}\left(\left|y_{\varepsilon}\right|\right) .
\end{aligned}
$$

But

$$
\begin{aligned}
& \sup _{(\zeta, \xi) \in \mathrm{FL}(O)}\left\{D\left(x \mapsto-\frac{\widetilde{d}_{\varepsilon}^{2}\left(x, y_{\varepsilon}\right)}{2 \varepsilon}\right)(O, \zeta)-\xi\right\} \\
& =\max \left(\max _{j \neq 2} H_{j}^{+}\left(\frac{k_{j} \widetilde{d}_{\varepsilon}\left(O, y_{\varepsilon}\right)}{\varepsilon}\right), H_{2}^{+}\left(-\frac{K_{2} \mu_{2} \varepsilon^{\eta_{2}} \widetilde{d}_{\varepsilon}\left(O, y_{\varepsilon}\right)\left|y_{\varepsilon}\right|^{\mu_{2}-1}}{\varepsilon}\right)\right)
\end{aligned}
$$

and the desired contradiction follows from (5.12).

3rd case: $x_{\varepsilon} \neq O$ and $y_{\varepsilon}=O$ : Assume for example that $x_{\varepsilon} \in \overline{J_{1}} \backslash\{O\}$ (we proceed similarly in the other cases). From (5.24) and (5.25), we get

$$
\begin{aligned}
& \lambda\left(u\left(x_{\varepsilon}\right)-v(O)\right) \\
& \leq-H_{1}\left(\frac{k_{1} \widetilde{d}_{\varepsilon}\left(x_{\varepsilon}, O\right)}{\varepsilon}\right)+\sup _{(\zeta, \xi) \in \mathrm{FL}(O)}\left\{-D\left(y \mapsto-\frac{\widetilde{d}_{\varepsilon}^{2}\left(x_{\varepsilon}, y\right)}{2 \varepsilon}\right)(O, \zeta)-\xi\right\} \\
& \quad+C \frac{\widetilde{d}_{\varepsilon}^{2}\left(x_{\varepsilon}, O\right)}{\varepsilon}+\omega_{\ell}\left(\left|x_{\varepsilon}\right|\right) .
\end{aligned}
$$


But

$$
\begin{aligned}
& \sup _{(\zeta, \xi) \in \mathrm{FL}(O)}\left\{-D\left(y \mapsto-\frac{\widetilde{d}_{\varepsilon}^{2}\left(x_{\varepsilon}, y\right)}{2 \varepsilon}\right)(O, \zeta)-\xi\right\} \\
& =\max \left(\max _{j \neq 1} H_{j}^{+}\left(D\left(y \mapsto-\frac{\widetilde{d}_{\varepsilon}^{2}\left(x_{\varepsilon}, y\right)}{2 \varepsilon}\right)\left(O, e_{j}\right)\right), H_{1}^{+}\left(\frac{k_{1} \widetilde{d}_{\varepsilon}\left(x_{\varepsilon}, O\right)}{\varepsilon}\right)\right) .
\end{aligned}
$$

We have $H_{i}^{+}(q) \leq H_{i}(q)$, for all $q$ and $i$. Thus, the desired contradiction follows from the identity

$$
\frac{k_{1} \widetilde{d}_{\varepsilon}\left(x_{\varepsilon}, O\right)}{\varepsilon}=D\left(x \mapsto \frac{\widetilde{d}_{\varepsilon}^{2}(x, O)}{2 \varepsilon}\right)\left(x_{\varepsilon}, e_{1}\right),
$$

and (5.21).

Theorem 5.7. Under all the assumptions made in Theorem 5.6, if $u$ and $v$ are respectively a subsolution of (4.1) in $\mathcal{G}$ and a supersolution of (4.1) in $\overline{\mathcal{G}}$ then $u \leq v$ in $\overline{\mathcal{G}}$.

The value function is the only constrained viscosity solution of (4.1) in $\overline{\mathcal{G}}$.

Proof. The proof resembles that of Theorem 5.6, with more technicalities near $\partial \mathcal{V}$, see [2, p. 278]. We skip it for brevity, but we only mention that it makes use of the piecewise linear vector field $\eta$ on $\overline{\mathcal{G}}$ defined by $\eta(x)=$ $-\left(4\left(x_{i}-1 / 4\right) 1_{1 / 4 \leq x_{i}<1 / 2}+1_{x_{i} \geq 1 / 2}\right) e_{i}$ in $\overline{J_{i}}$, which plays the role of the vector field $\eta$ in the formula (5.21) in [2, p. 278].

Remark 5.5. Here, we briefly sketch how the function $\tilde{d}_{\varepsilon}$ should be modified in order to extend Theorem 5.6 to more general networks with a finite number of crosspoints $\left(O_{i}\right)_{1 \leq i \leq P}$.

The edges are still denoted $\left(J_{i}\right)_{1 \leq i \leq N}$. Let $\tilde{d}_{\ell, \varepsilon}(x, y)$ be the function defined by (5.20) for $x$ and $y$ in the union of the edges adjacent to $O_{\ell}$. Let $J_{i}$ join two crosspoints $O_{i, 1}=O_{j}$ and $O_{i, 2}=O_{k}$ (the order is arbitrary): we define $\bar{d}_{i, \varepsilon}(x, y)$ on $\bar{J}_{i} \times \bar{J}_{i}$ by

$$
\bar{d}_{i, \varepsilon}(x, y)=\varphi_{i}(x, y) \tilde{d}_{j, \varepsilon}(x, y)+\left(1-\varphi_{i}(x, y)\right) \tilde{d}_{k, \varepsilon}(x, y),
$$

where $\varphi_{i}(x, y)$ is a smooth function such that

$$
\begin{aligned}
\varphi_{i}(x, y)=1 & \text { if }(x, y) \in\left[O_{i, 1}, O_{i, 1}+1 / 3\left(O_{i, 2}-O_{i, 1}\right)\right]^{2}, \\
\varphi_{i}(x, y)=0 & \text { if }(x, y) \in\left[O_{i, 2}+1 / 3\left(O_{i, 1}-O_{i, 2}\right), O_{i, 2}\right]^{2}, \\
0 \leq \varphi_{i}(x, y) \leq 1 & \text { in } \bar{J}_{i}^{2} .
\end{aligned}
$$

If only one endpoint of $J_{i}$ is a crosspoint, say $O_{j}$, then $\bar{d}_{i, \varepsilon}(x, y)=\tilde{d}_{j, \varepsilon}(x, y)$ for $(x, y) \in \bar{J}_{i}^{2}$. Note that $\bar{d}_{i, \varepsilon}(x, y) \neq \bar{d}_{i, \varepsilon}(y, x)$.

Let $t \mapsto \gamma(t)$ be a path from $x$ to $y$ on $\overline{\mathcal{G}}$, such that the velocity $\gamma^{\prime}(t)$ can vary only if $\gamma(t)$ is at a crosspoint. Let $\left(i_{n}\right)_{0, \ldots, N_{\gamma}}$ be the indices of the edges successively crossed by $\gamma$. Note that if $N_{\gamma}>1$ then the whole edges $J_{i_{1}}, \ldots, J_{i_{N_{\gamma}-1}}$ are crossed. Assume also that $\gamma$ crosses $\left(O_{i_{j}}\right)_{1 \leq j \leq N_{\gamma}-1}$ in that order: $O_{i_{j}}$ and $O_{i_{j+1}}$ are the endpoints of $J_{i_{j}}$. We define $|\gamma|_{\varepsilon}=\bar{d}_{i_{0}, \varepsilon}\left(x, O_{i_{1}}\right)+$ 
$\sum_{j=1}^{N_{\gamma}-1} \bar{d}_{i_{j}, \varepsilon}\left(O_{i_{j}}, O_{i_{j+1}}\right)+\bar{d}_{i_{N_{\gamma}, \varepsilon}}\left(O_{i_{N_{\gamma}}}, y\right)$, if $N_{\gamma} \geq 1$ and $|\gamma|_{\varepsilon}=\bar{d}_{i_{0}, \varepsilon}(x, y)$ otherwise. Finally, we define $\bar{d}_{\varepsilon}(x, y)=\min _{\text {paths }} \gamma|\gamma|_{\varepsilon}$.

It is possible to check from the fact that the exponents $\eta_{j}$ in (5.20) are strictly less than $1 / 2$, [see (5.22)], that if $\bar{d}_{\varepsilon}\left(x_{\varepsilon}, y_{\varepsilon}\right) \leq C \sqrt{\varepsilon}$, then $x_{\varepsilon}$ and $y_{\varepsilon}$ lie either on the same edge or belong to two different edges adjacent to a same crosspoint, so near $\left(x_{\varepsilon}, y_{\varepsilon}\right), \bar{d}_{\varepsilon}^{2}$ is regular in the sense of Definition 3.1. The function $\bar{d}_{\varepsilon}(x, y)$ can thus be used in place of $\tilde{d}_{\varepsilon}(x, y)$ for extending Theorem 5.6 to the case of a network made of a finite number of straight edges.

Remark 5.6. (Multivalued optimal feedback) The comparison principle has many consequences: one of them is the existence of multivalued optimal feedback under suitable assumptions. We refer to [2] and references therein for a complete introduction to this topic. Here, for brevity, we just give an example of a result that can be obtained. Knowing the value function $v$, let us introduce the sets of controls:

$$
S_{D}(x)=\left\{a \in A_{x}, \liminf _{t \rightarrow 0^{+}} \frac{v(x+t f(x, a))-v(x)}{t}+\ell(x, a) \leq \lambda v(x)\right\} .
$$

If the comparison principle holds, if the value function is Lipschitz continuous, and if for each $x \in \overline{\mathcal{G}}$, there exists an optimal control, then the multivalued feedback $S_{D}$ is fully optimal. This means that for all $x \in \overline{\mathcal{G}}$, every solution of

$$
\begin{cases}y(t)=x+\int_{0}^{t} f(y(s), \alpha(s)) d s, & t \geq 0, \\ \alpha(s) \in S_{D}(y(s)), & \text { for a.a. } s>0,\end{cases}
$$

is optimal. The proof is very much alike that given in [2, Section III.2.5, see in particular Theorem III.2.61].

\section{Acknowledgments}

This work was partially done when Fabio Camilli was visiting University ParisDiderot. This work was partially done when Alessandra Cutrì was visiting the University of Rennes 1 with the support of IRMAR. The work was partially supported by the PRIN MIUR 2009: Metodi di viscosità, geometrici e di controllo per modelli diffusivi nonlineari.

\section{Appendix A. Proof of Proposition 4.1}

Take first $x \in \mathcal{G} \backslash\{O\}$. In this case, the proof is standard (see [2], Lemma 2.41, p. 129), but we give it for completeness.

We can assume that $x \in J_{1}$. The inclusion $\mathrm{FL}(x) \subset \widetilde{f} \ell(x)$ is obtained as follows: take $\zeta=\sum_{j=1}^{J} \mu_{j} f\left(x, a_{j}\right), \xi=\sum_{j=1}^{J} \mu_{j} \ell\left(x, a_{j}\right)$ with $a_{j} \in A_{x}$ and $\sum_{j} \mu_{j}=1,0 \leq \mu_{j}$. For $t_{n}$ small enough, it is possible to construct a control $\alpha_{n} \in \mathcal{A}_{x}$ such that $\alpha_{n}(t)=a_{j}$ for $\left(\sum_{k<j} \mu_{k}\right) t_{n}<t \leq\left(\sum_{k \leq j} \mu_{k}\right) t_{n}$ : we have $\frac{1}{t_{n}} \int_{0}^{t_{n}} f\left(y\left(t ; x, \alpha_{n}\right), \alpha_{n}(t)\right) d t=\frac{1}{t_{n}} \int_{0}^{t_{n}} f\left(x, \alpha_{n}(t)\right) d t+o(1)=\sum_{j} \mu_{j} f\left(x, a_{j}\right)+$ 
$o(1)$, so

$$
\lim _{n \rightarrow \infty} \frac{1}{t_{n}} \int_{0}^{t_{n}} f\left(y\left(t ; x, \alpha_{n}\right), \alpha_{n}(t)\right) d t=\zeta .
$$

Similarly,

$$
\lim _{n \rightarrow \infty} \frac{1}{t_{n}} \int_{0}^{t_{n}} \ell\left(y\left(t ; x, \alpha_{n}\right), \alpha_{n}(t)\right) d t=\xi .
$$

Finally, for $(\zeta, \xi) \in \mathrm{FL}(x)$, we approximate $(\zeta, \xi)$ by $\left(\zeta_{m}, \xi_{m}\right)_{m \in \mathbb{N}}$, where $\left(\zeta_{m}, \xi_{m}\right)$ is a convex combination of $(f(x, a), \ell(x, a)), a \in A_{x}$, and we conclude by a diagonal process.

For the opposite inclusion, since $x \in \mathcal{G} \backslash\{O\}$, we know from (2.7) that there exists $\tau>0$, such that for all $\alpha \in \mathcal{A}_{x}, \alpha(t) \in A_{x}$ for $0 \leq t<\tau$. Therefore,

$$
\left(\frac{1}{s} \int_{0}^{s} f(x, \alpha(t)) d t, \frac{1}{s} \int_{0}^{s} \ell(x, \alpha(t)) d t\right) \in \mathrm{FL}(x)
$$

for $s$ small enough. This and the continuity of $f$ and $\ell$ w.r.t. their first argument imply that $\widetilde{f} \ell(x) \subset \mathrm{FL}(x)$. We have proved (4.4).

We now consider $x=O$. We first discuss the inclusion $\operatorname{FL}(O) \subset \widetilde{f} \ell(O)$ : we take $\zeta=\sum_{j=1}^{J} \mu_{j} f\left(O, a_{j}\right), \xi=\sum_{j=1}^{J} \mu_{j} \ell\left(O, a_{j}\right)$ with $a_{j} \in A^{1}$ and we assume that $\zeta \in \mathbb{R}_{+} e_{1}$. Up to a permutation of the indices, it is possible to assume that there exists $J^{\prime}, 1<J^{\prime} \leq J$ such that $f\left(O, a_{j}\right) \in \mathbb{R}_{+} e_{1}$ for $j \leq J^{\prime}$ and that $f\left(O, a_{j}\right) \in \mathbb{R}_{-} e_{1}$ for $j>J^{\prime}$. Then by a similar argument as above, $(\zeta, \xi) \in$ $\widetilde{f} \ell(O)$. By a diagonal process, this implies that

$$
\overline{\mathrm{co}}\left((f(O, a), \ell(O, a)): a \in A^{1}\right) \cap\left(\mathbb{R}_{+} e_{1} \times \mathbb{R}\right) \subset \tilde{f} \ell(O) .
$$

Similarly $\overline{\mathrm{co}}\left((f(O, a), \ell(O, a)): a \in A^{j}\right) \cap\left(\mathbb{R}_{+} e_{j} \times \mathbb{R}\right) \subset \widetilde{f \ell}(O)$, so we have proved (4.5).

The proof of (4.6) is similar.

To prove points (b 1) and (b 2), we consider $\zeta \in \tilde{f}(O)$ and make out two cases:

- $\zeta=0$ : from Assumption 2.2, $\mathrm{FL}(O) \cap(\{0\} \times \mathbb{R}) \neq \emptyset$.

From Assumption 2.3, $\min \{\xi:(0, \xi) \in \mathrm{FL}(O)\}=m_{O}$.

On the other hand, for all sequences $t_{n} \rightarrow 0^{+}$and $\alpha_{n} \in \mathcal{A}_{O}$,

$$
\liminf _{n \rightarrow \infty} \frac{1}{t_{n}} \int_{0}^{t_{n}} \ell\left(y\left(t ; O, \alpha_{n}\right), \alpha_{n}(t)\right) d t \geq m_{O} .
$$

Therefore,

$$
\min \{\xi:(0, \xi) \in \operatorname{FL}(O)\} \leq \min \{\xi:(0, \xi) \in \widetilde{f} \ell(O)\},
$$

and this inequality is in fact an identity, because $\mathrm{FL}(O) \subset \tilde{f} \ell(O)$.

- $\zeta \neq 0$ : we can suppose that $0 \neq \zeta \in \mathbb{R}_{+} e_{1}$. There exist sequences $\alpha_{n} \in \mathcal{A}_{O}$ and $t_{n}>0$ such that $t_{n} \rightarrow 0^{+}, \lim _{n \rightarrow \infty} \frac{1}{t_{n}} \int_{0}^{t_{n}} f\left(y\left(t ; O, \alpha_{n}\right), \alpha_{n}(t)\right) d t=\zeta$. Up to an extraction, we may assume that $\lim _{n \rightarrow \infty} \frac{1}{t_{n}} \int_{0}^{t_{n}} \ell\left(y\left(t ; O, \alpha_{n}\right)\right.$, $\left.\alpha_{n}(t)\right) d t=\mu$. 
Since $0 \neq \zeta \in \mathbb{R}_{+} e_{1}$, there exists $s_{n}, 0 \leq s_{n}<t_{n}$ such that $y\left(s_{n} ; O, \alpha_{n}\right)=$ $O$ and $y\left(t ; O, \alpha_{n}\right) \in J_{1}$ for all $t, s_{n}<t \leq t_{n}$. From (2.7), this implies that $\alpha_{n}(t) \in A^{1}$ for all $t, s_{n}<t<t_{n}$. Hence,

$$
\begin{aligned}
& \left(\frac{1}{t_{n}-s_{n}} \int_{s_{n}}^{t_{n}} f\left(O, \alpha_{n}(t)\right) d t, \frac{1}{t_{n}-s_{n}} \int_{s_{n}}^{t_{n}} \ell\left(O, \alpha_{n}(t)\right) d t\right) \\
& \quad \in \overline{\mathrm{co}}\left((f(O, a), \ell(O, a)): a \in A^{1}\right) \cap\left(\mathbb{R}_{+} e_{1} \times \mathbb{R}\right) .
\end{aligned}
$$

Therefore, since $\left(0, m_{O}\right) \in \overline{\mathrm{co}}\left((f(O, a), \ell(O, a)): a \in A^{1}\right)$ from Assumption 2.3 , we get that

$$
\begin{aligned}
& \left(\frac{1}{t_{n}} \int_{s_{n}}^{t_{n}} f\left(O, \alpha_{n}(t)\right) d t, \frac{1}{t_{n}} \int_{s_{n}}^{t_{n}} \ell\left(O, \alpha_{n}(t)\right) d t+\frac{s_{n}}{t_{n}} m_{O}\right) \\
& \in \overline{\operatorname{co}}\left((f(O, a), \ell(O, a)): a \in A^{1}\right) \cap\left(\mathbb{R}_{+} e_{1} \times \mathbb{R}\right) .
\end{aligned}
$$

Up to the extraction of a subsequence, we may say that $\frac{1}{t_{n}} \int_{s_{n}}^{t_{n}} \ell\left(O, \alpha_{n}(t)\right) d t+\frac{s_{n}}{t_{n}} m_{O}$ converges to a real number $\xi$. Moreover, from the continuity of $f$,

$\zeta=\lim _{n \rightarrow \infty} \frac{1}{t_{n}} \int_{s_{n}}^{t_{n}} f\left(y\left(t ; O, \alpha_{n}\right), \alpha_{n}(t)\right) d t=\lim _{n \rightarrow \infty} \frac{1}{t_{n}} \int_{s_{n}}^{t_{n}} f\left(O, \alpha_{n}(t)\right) d t$, and we see that $(\zeta, \xi) \in \mathrm{FL}(O) \cap\left(\mathbb{R}_{+} e_{1} \times \mathbb{R}\right)$, which proves point (b 1$)$. We also see that

$$
\xi \leq \lim \frac{1}{t_{n}} \int_{0}^{t_{n}} \ell\left(O, \alpha_{n}(t)\right) d t=\mu
$$

where the last identity comes from the continuity of $\ell$. We have proved point (b 2), since $\xi \leq \mu$ is true for all $\mu$ such that $(\zeta, \mu) \in \widetilde{f} \ell(O)$.

\section{Appendix B. Proof of Lemma 5.4}

Since $m_{O}$ plays no role in the proof, we may assume that $m_{O}=0$.

We are going to prove that it is enough to choose the constants $k_{j}$ and $K_{j}$ such that

1. If there does not exist $i \in\{1, \ldots, N\}$ such that $\alpha_{i}=\gamma_{k}$ for all $k=$ $1, \ldots, N$,

$$
k_{j} \geq \max _{i}\left(\frac{\mu_{i}\left(a_{i}\left(\zeta_{i}^{-}\right)^{\alpha_{i}}+d_{j} \zeta_{j}^{+}\right)}{d_{j} \zeta_{i}^{-}} K_{i}, \frac{\mu_{i} b_{i}}{d_{j}} K_{i}\right), \quad \forall j=1, \ldots, N .
$$

2. In the opposite case, let $\gamma$ be the common value of the $\gamma_{k}$ : then, $\forall j=$ $1, \ldots, N$,

$k_{j} \geq \max \left(\max _{i}\left(\frac{\mu_{i}\left(a_{i}\left(\zeta_{i}^{-}\right)^{\alpha_{i}}+d_{j} \zeta_{j}^{+}\right)}{d_{j} \zeta_{i}^{-}} K_{i}, \frac{\mu_{i} b_{i}}{d_{j}} K_{i}\right), \max _{i: \alpha_{i}=\gamma} K_{i}\left(\frac{a_{i}}{c_{j}}\right)^{1 / \gamma}\right)$. 
Consider a sequence $\left(x_{\varepsilon}, y_{\varepsilon}\right)$ such that $x_{\varepsilon} \in \overline{J_{1}}$ and $y_{\varepsilon} \in \overline{J_{2}}$ and $\lim _{\varepsilon \rightarrow 0} \frac{\tilde{d}_{\varepsilon}^{2}\left(x_{\varepsilon}, y_{\varepsilon}\right)}{\varepsilon}=0$ : it is clear from the observations above that $\lim _{\varepsilon \rightarrow 0} x_{\varepsilon}=\lim _{\varepsilon \rightarrow 0} y_{\varepsilon}=O$.

We make out two cases:

Case 1. $\max \left(\eta_{2}, \mu_{2}-1\right)>0$. In this case, $\lim _{\varepsilon \rightarrow 0} \varepsilon^{\eta_{2}}\left|y_{\varepsilon}\right|^{\mu_{2}-1}=0$. With $k_{1}$ and $K_{2}$ fixed such that (5.27) holds, we can take $\varepsilon$ small enough such that $\frac{k_{1} \tilde{d}_{\varepsilon}\left(x_{\varepsilon}, y_{\varepsilon}\right)}{\varepsilon} \leq \zeta_{1}^{+}$implies that $\frac{\mu_{2} K_{2} \varepsilon^{\eta_{2}}\left|y_{\varepsilon}\right|^{\mu_{2}-1} \tilde{d}_{\varepsilon}\left(x_{\varepsilon}, y_{\varepsilon}\right)}{\varepsilon} \leq \zeta_{2}^{-}$: therefore, up to subsequence extraction, we can make out three subcases

Subcase 1. $\frac{k_{1} \tilde{d}_{\varepsilon}\left(x_{\varepsilon}, y_{\varepsilon}\right)}{\varepsilon} \leq \zeta_{1}^{+}$and $\frac{\mu_{2} K_{2} \varepsilon^{\eta_{2}}\left|y_{\varepsilon}\right|^{\mu_{2}-1} \tilde{d}_{\varepsilon}\left(x_{\varepsilon}, y_{\varepsilon}\right)}{\varepsilon} \leq \zeta_{2}^{-}$: we have

$$
\begin{aligned}
H_{1}\left(D\left(x \mapsto \frac{\tilde{d}_{\varepsilon}^{2}\left(x, y_{\varepsilon}\right)}{2 \varepsilon}\right)\left(x_{\varepsilon}, e_{1}\right)\right) & \geq c_{1}\left(k_{1} \frac{\tilde{d}_{\varepsilon}\left(x_{\varepsilon}, y_{\varepsilon}\right)}{\varepsilon}\right)^{\gamma_{1}}, \\
H_{2}\left(D\left(y \mapsto-\frac{\tilde{d}_{\varepsilon}^{2}\left(x_{\varepsilon}, y\right)}{2 \varepsilon}\right)\left(y_{\varepsilon}, e_{2}\right)\right) & \leq a_{2}\left(\frac{\mu_{2} K_{2} \varepsilon^{\eta_{2}}\left|y_{\varepsilon}\right|^{\mu_{2}-1} \tilde{d}_{\varepsilon}\left(x_{\varepsilon}, y_{\varepsilon}\right)}{\varepsilon}\right)^{\alpha_{2}} .
\end{aligned}
$$

Therefore

$$
H_{1}\left(D\left(x \mapsto \frac{\tilde{d}_{\varepsilon}^{2}\left(x, y_{\varepsilon}\right)}{2 \varepsilon}\right)\left(x_{\varepsilon}, e_{1}\right)\right) \geq H_{2}\left(D\left(y \mapsto-\frac{\tilde{d}_{\varepsilon}^{2}\left(x_{\varepsilon}, y\right)}{2 \varepsilon}\right)\left(y_{\varepsilon}, e_{2}\right)\right)
$$

will be true if

$$
c_{1} k_{1}^{\gamma_{1}} \geq a_{2}\left(\mu_{2} K_{2}\right)^{\alpha_{2}} \varepsilon^{\alpha_{2} \eta_{2}+\gamma_{1}-\alpha_{2}}\left|y_{\varepsilon}\right|^{\alpha_{2}\left(\mu_{2}-1\right)} \tilde{d}_{\varepsilon}\left(x_{\varepsilon}, y_{\varepsilon}\right)^{\alpha_{2}-\gamma_{1}} .
$$

1. If $\gamma_{1} \geq \alpha_{2}$, then $\tilde{d}_{\varepsilon}\left(x_{\varepsilon}, y_{\varepsilon}\right)^{\alpha_{2}-\gamma_{1}} \leq\left(K_{2} \varepsilon^{\eta_{2}}\left|y_{\varepsilon}\right|^{\mu_{2}}\right)^{\alpha_{2}-\gamma_{1}}$, so a sufficient condition for (5.29) is that

$$
\begin{aligned}
c_{1} k_{1}^{\gamma_{1}} & \geq a_{2} \mu_{2}^{\alpha_{2}} K_{2}^{2 \alpha_{2}-\gamma_{1}} \varepsilon^{\left(2 \alpha_{2}-\gamma_{1}\right) \eta_{2}+\gamma_{1}-\alpha_{2}}\left|y_{\varepsilon}\right|^{\left(2 \alpha_{2}-\gamma_{1}\right) \mu_{2}-\alpha_{2}} \\
& =a_{2} \mu_{2}^{\alpha_{2}} K_{2}^{2 \alpha_{2}-\gamma_{1}} \varepsilon^{\alpha_{2} \eta_{2}} \varepsilon^{\left(\alpha_{2}-\gamma_{1}\right)\left(\eta_{2}-1\right)}\left|y_{\varepsilon}\right|^{\left(2 \alpha_{2}-\gamma_{1}\right) \mu_{2}-\alpha_{2}}
\end{aligned}
$$

But $\mu_{2} \geq \alpha_{2} /\left(2 \alpha_{2}-\gamma_{1}\right)$ and $0 \leq \eta_{2}<1 / 2$. Therefore, the three exponents in the right hand side of (5.30), namely $\alpha_{2} \eta_{2},\left(\alpha_{2}-\gamma_{1}\right)\left(\eta_{2}-1\right)$ and $\left(2 \alpha_{2}-\gamma_{1}\right) \mu_{2}-\alpha_{2}$ are nonnegative. Moreover they cannot vanish at the same time, because it would imply that $\eta_{2}=0, \mu_{2}=\alpha_{2} /\left(2 \alpha_{2}-\gamma_{1}\right), \gamma_{1}=$ $\alpha_{2}$ and then $\mu_{2}=1$, which would contradict $\max \left(\eta_{2}, \mu_{2}-1\right)>0$. Hence, having fixed $k_{j}$ and $K_{j}$ satisfying $(5.27)$, (5.29) is obtained as soon as $\varepsilon$ is small enough.

2. If $\gamma_{1}<\alpha_{2}$, then $\lim \tilde{d}_{\varepsilon}^{\alpha_{2}-\gamma_{1}}\left(x_{\varepsilon}, y_{\varepsilon}\right)=0$. Moreover, there exists a constant $C$ such that, for small values of $\varepsilon$,

$$
a_{2}\left(\mu_{2} K_{2}\right)^{\alpha_{2}} \varepsilon^{\alpha_{2} \eta_{2}+\gamma_{1}-\alpha_{2}}\left|y_{\varepsilon}\right|^{\alpha_{2}\left(\mu_{2}-1\right)} \leq C \varepsilon^{\alpha_{2} \eta_{2}+\gamma_{1}-\alpha_{2}} .
$$

Since $\eta_{2} \geq\left(\alpha_{2}-\gamma_{1}\right) / \alpha_{2}$ and $\lim \tilde{d}_{\varepsilon}^{\alpha_{2}-\gamma_{1}}\left(x_{\varepsilon}, y_{\varepsilon}\right)=0$, the right hand side of (5.29) tends to zero as $\varepsilon$ tends to zero. To get (5.29), it is enough to take $\varepsilon$ small enough. 
Subcase 2. $\frac{k_{1} \tilde{d}_{\varepsilon}\left(x_{\varepsilon}, y_{\varepsilon}\right)}{\varepsilon}>\zeta_{1}^{+}$and $\frac{\mu_{2} K_{2} \varepsilon^{\eta_{2}}\left|y_{\varepsilon}\right|^{\mu_{2}-1} \tilde{d}_{\varepsilon}\left(x_{\varepsilon}, y_{\varepsilon}\right)}{\varepsilon} \leq \zeta_{2}^{-}$: we have

$$
\begin{gathered}
H_{1}\left(D\left(x \mapsto \frac{\tilde{d}_{\varepsilon}^{2}\left(x, y_{\varepsilon}\right)}{2 \varepsilon}\right)\left(x_{\varepsilon}, e_{1}\right)\right) \geq d_{1}\left(k_{1} \frac{\tilde{d}_{\varepsilon}\left(x_{\varepsilon}, y_{\varepsilon}\right)}{\varepsilon}-\zeta_{1}^{+}\right)+c_{1}\left(\zeta_{1}^{+}\right)^{\gamma_{1}}, \\
H_{2}\left(D\left(y \mapsto-\frac{\tilde{d}_{\varepsilon}^{2}\left(x_{\varepsilon}, y\right)}{2 \varepsilon}\right)\left(y_{\varepsilon}, e_{2}\right)\right) \leq a_{2}\left(\frac{\mu_{2} K_{2} \varepsilon^{\eta_{2}}\left|y_{\varepsilon}\right|^{\mu_{2}-1} \tilde{d}_{\varepsilon}\left(x_{\varepsilon}, y_{\varepsilon}\right)}{\varepsilon}\right)^{\alpha_{2}} .
\end{gathered}
$$

Let us define $q$ by $q=\frac{\mu_{2} K_{2} \varepsilon^{\eta_{2}}\left|y_{\varepsilon}\right|^{\mu_{2}-1} \tilde{d}_{\varepsilon}\left(x_{\varepsilon}, y_{\varepsilon}\right)}{\varepsilon}$. We have $\frac{\tilde{d}_{\varepsilon}\left(x_{\varepsilon}, y_{\varepsilon}\right)}{\varepsilon}=$ $\frac{\varepsilon^{-\eta_{2}}\left|y_{\varepsilon}\right|^{1-\mu_{2}} q}{\mu_{2} K_{2}}$. We wish to prove that

$$
d_{1}\left(k_{1} \frac{\varepsilon^{-\eta_{2}}\left|y_{\varepsilon}\right|^{1-\mu_{2}} q}{\mu_{2} K_{2}}-\zeta_{1}^{+}\right)+c_{1}\left(\zeta_{1}^{+}\right)^{\gamma_{1}} \geq a_{2} q^{\alpha_{2}},
$$

for all $q$ such that $\frac{\mu_{2} K_{2} \zeta_{1}^{+}}{k_{1}} \varepsilon^{\eta_{2}}\left|y_{\varepsilon}\right|^{\mu_{2}-1} \leq q \leq \zeta_{2}^{-}$. Note that this interval is non empty if $\varepsilon$ is small enough because $\lim _{\varepsilon \rightarrow 0} \varepsilon^{\eta_{2}}\left|y_{\varepsilon}\right|^{\mu_{2}-1}=0$.

For $q=\frac{\mu_{2} K_{2} \zeta_{1}^{+}}{k_{1}} \varepsilon^{\eta_{2}}\left|y_{\varepsilon}\right|^{\mu_{2}-1}$, we have already seen in the subcase 1 that (5.31) is true provided $\varepsilon$ is small enough. To be more precise, we have $d_{1}\left(k_{1} \frac{\varepsilon^{-\eta_{2}}\left|y_{\varepsilon}\right|^{1-\mu_{2}} q}{\mu_{2} K_{2}}-\zeta_{1}^{+}\right)+c_{1}\left(\zeta_{1}^{+}\right)^{\gamma_{1}} \geq c_{1}\left(\zeta_{1}^{+}\right)^{\gamma_{1}}$ which is larger than $a_{2} q^{\alpha_{2}}=$ $a_{2}\left(\frac{\mu_{2} K_{2} \zeta_{1}^{+}}{k_{1}} \varepsilon^{\eta_{2}}\left|y_{\varepsilon}\right|^{\mu_{2}-1}\right)^{\alpha_{2}}$ for $\varepsilon$ small enough.

By the convexity of $q \mapsto a_{2} q^{\alpha_{2}}$ and the linearity of $q \mapsto d_{1}\left(k_{1} \frac{\varepsilon^{-\eta_{2}}\left|y_{\varepsilon}\right|^{1-\mu_{2}} q}{\mu_{2} K_{2}}\right.$ $\left.-\zeta_{1}^{+}\right)+c_{1}\left(\zeta_{1}^{+}\right)^{\gamma_{1}}$, it is then enough to prove (5.31) for $q=\zeta_{2}^{-}$, i.e. that

$$
d_{1}\left(k_{1} \frac{\varepsilon^{-\eta_{2}}\left|y_{\varepsilon}\right|^{1-\mu_{2}} \zeta_{2}^{-}}{\mu_{2} K_{2}}-\zeta_{1}^{+}\right)+c_{1}\left(\zeta_{1}^{+}\right)^{\gamma_{1}} \geq a_{2}\left(\zeta_{2}^{-}\right)^{\alpha_{2}} \text {. }
$$

But for $\varepsilon \leq 1$, since $\left|y_{\varepsilon}\right| \leq 1$, (5.32) is a consequence of the condition:

$$
d_{1}\left(k_{1} \frac{\zeta_{2}^{-}}{\mu_{2} K_{2}}-\zeta_{1}^{+}\right) \geq a_{2}\left(\zeta_{2}^{-}\right)^{\alpha_{2}},
$$

which is implied by (5.27).

Subcase 3. $\frac{k_{1} \tilde{d}_{\varepsilon}\left(x_{\varepsilon}, y_{\varepsilon}\right)}{\varepsilon}>\zeta_{1}^{+}$and $\frac{\mu_{2} K_{2} \varepsilon^{\eta_{2}}\left|y_{\varepsilon}\right|^{\mu_{2}-1} \tilde{d}_{\varepsilon}\left(x_{\varepsilon}, y_{\varepsilon}\right)}{\varepsilon} \geq \zeta_{2}^{-}$: we have

$$
\begin{aligned}
H_{1}\left(D\left(x \mapsto \frac{\tilde{d}_{\varepsilon}^{2}\left(x, y_{\varepsilon}\right)}{2 \varepsilon}\right)\left(x_{\varepsilon}, e_{1}\right)\right) \geq & d_{1}\left(k_{1} \frac{\tilde{d}_{\varepsilon}\left(x_{\varepsilon}, y_{\varepsilon}\right)}{\varepsilon}-\zeta_{1}^{+}\right)+c_{1}\left(\zeta_{1}^{+}\right)^{\gamma_{1}}, \\
H_{2}\left(D\left(y \mapsto-\frac{\tilde{d}_{\varepsilon}^{2}\left(x_{\varepsilon}, y\right)}{2 \varepsilon}\right)\left(y_{\varepsilon}, e_{2}\right)\right) \leq & b_{2}\left(\frac{\mu_{2} K_{2} \varepsilon^{\eta_{2}}\left|y_{\varepsilon}\right|^{\mu_{2}-1} \tilde{d}_{\varepsilon}\left(x_{\varepsilon}, y_{\varepsilon}\right)}{\varepsilon}-\zeta_{2}^{-}\right) \\
& +a_{2}\left(\zeta_{2}^{-}\right)^{\alpha_{2}} .
\end{aligned}
$$

We set $q=\frac{\tilde{d}_{\varepsilon}\left(x_{\varepsilon}, y_{\varepsilon}\right)}{\varepsilon}: q$ takes its values in $\left[\frac{\zeta_{2}^{-}}{\mu_{2} K_{2}} \varepsilon^{-\eta_{2}}\left|y_{\varepsilon}\right|^{1-\mu_{2}},+\infty\right)$. We wish to show that for $q$ in this interval,

$$
d_{1}\left(k_{1} q-\zeta_{1}^{+}\right)+c_{1}\left(\zeta_{1}^{+}\right)^{\gamma_{1}} \geq b_{2}\left(\mu_{2} K_{2} \varepsilon^{\eta_{2}}\left|y_{\varepsilon}\right|^{\mu_{2}-1} q-\zeta_{2}^{-}\right)+a_{2}\left(\zeta_{2}^{-}\right)^{\alpha_{2}} .
$$

In Subcase 2, we have already seen that (5.33) holds for $q=\frac{\zeta_{2}^{-}}{\mu_{2} K_{2}} \varepsilon^{-\eta_{2}}\left|y_{\varepsilon}\right|^{1-\mu_{2}}$. 
The desired inequality (5.33) will hold in the whole half-line if furthermore $d_{1} k_{1} \geq b_{2} \mu_{2} K_{2} \varepsilon^{\eta_{2}}\left|y_{\varepsilon}\right|^{\mu_{2}-1}$. This is a consequence of $d_{1} k_{1} \geq b_{2} \mu_{2} K_{2}$, which is implied by (5.27).

Case 2. $\max \left(\eta_{2}, \mu_{2}-1\right)=0$. From $\eta_{2}=0$, we deduce that $\alpha_{2} \leq \min _{k} \gamma_{k}$ and from $\mu_{2}=1$ we deduce that $\max _{k} \gamma_{k} \leq \alpha_{2}$. This implies that for all $k, \gamma_{k}=\alpha_{2}$. Let $\gamma=\alpha_{2}$ be the common value of the $\gamma_{k}$. Thus the constants $k_{i}, K_{j}$ are chosen such that (5.28) holds.

We are led to comparing $H_{1}\left(\frac{k_{1} \tilde{d}_{\varepsilon}\left(x_{\varepsilon}, y_{\varepsilon}\right)}{\varepsilon}\right)$ and $H_{2}\left(-\frac{K_{2} \tilde{d}_{\varepsilon}\left(x_{\varepsilon}, y_{\varepsilon}\right)}{\varepsilon}\right)$, where

$$
H_{2}(-p) \leq \begin{cases}a_{2} p^{\alpha_{2}}, & 0 \leq p \leq \zeta_{2}^{-}, \\ b_{2}\left(p-\zeta_{2}^{-}\right)+a_{2}\left(\zeta_{2}^{-}\right)^{\alpha_{2}}, & p \geq \zeta_{2}^{-},\end{cases}
$$

and

$$
H_{1}(p) \geq \begin{cases}c_{1} p^{\alpha_{2}}, & 0 \leq p \leq \zeta_{1}^{+}, \\ d_{1}\left(p-\zeta_{1}^{+}\right)+c_{1}\left(\zeta_{1}^{+}\right)^{\alpha_{2}}, & p \geq \zeta_{1}^{+} .\end{cases}
$$

Up to the extraction of a subsequence, we can make out three cases:

Subcase 1. $\frac{k_{1} \tilde{d}_{\varepsilon}\left(x_{\varepsilon}, y_{\varepsilon}\right)}{\varepsilon} \leq \zeta_{1}^{+}$: from (5.28), we know that $k_{1} \geq \frac{\zeta_{1}^{+}}{\zeta_{2}^{-}} K_{2}$ which implies that $\frac{K_{2} \tilde{d}_{\varepsilon}\left(x_{\varepsilon}, y_{\varepsilon}\right)}{\varepsilon} \leq \zeta_{2}^{-}$. We have to compare $c_{1}\left(\frac{k_{1} \tilde{d}_{\varepsilon}\left(x_{\varepsilon}, y_{\varepsilon}\right)}{\varepsilon}\right)^{\alpha_{2}}$ and $a_{2}\left(\frac{K_{2} \tilde{d}_{\varepsilon}\left(x_{\varepsilon}, y_{\varepsilon}\right)}{\varepsilon}\right)^{\alpha_{2}}$. From $(5.28)$, we know that $k_{1} \geq\left(\frac{a_{2}}{c_{1}}\right)^{\frac{1}{\alpha_{2}}} K_{2}$, which implies that

$$
c_{1}\left(\frac{k_{1} \tilde{d}_{\varepsilon}\left(x_{\varepsilon}, y_{\varepsilon}\right)}{\varepsilon}\right)^{\alpha_{2}} \geq a_{2}\left(\frac{K_{2} \tilde{d}_{\varepsilon}\left(x_{\varepsilon}, y_{\varepsilon}\right)}{\varepsilon}\right)^{\alpha_{2}} .
$$

Subcase 2. $\frac{\zeta_{1}^{+}}{k_{1}} \leq \frac{\tilde{d}_{\varepsilon}\left(x_{\varepsilon}, y_{\varepsilon}\right)}{\varepsilon} \leq \frac{\zeta_{2}^{-}}{K_{2}}$ : we wish to prove that

$$
d_{1}\left(\frac{k_{1} \tilde{d}_{\varepsilon}\left(x_{\varepsilon}, y_{\varepsilon}\right)}{\varepsilon}-\zeta_{1}^{+}\right)+c_{1}\left(\zeta_{1}^{+}\right)^{\alpha_{2}} \geq a_{2}\left(\frac{K_{2} \tilde{d}_{\varepsilon}\left(x_{\varepsilon}, y_{\varepsilon}\right)}{\varepsilon}\right)^{\alpha_{2}}
$$

We have already seen that the inequality is true for $\frac{\tilde{d}_{\varepsilon}\left(x_{\varepsilon}, y_{\varepsilon}\right)}{\varepsilon}=\frac{\zeta_{1}^{+}}{k_{1}}$. By convexity, it is enough to prove the inequality for $\frac{\tilde{d}_{\varepsilon}\left(x_{\varepsilon}, y_{\varepsilon}\right)}{\varepsilon}=\frac{\zeta_{2}^{-}}{K_{2}}$. But from (5.28), $k_{1} \geq \frac{K_{2}}{\zeta_{2}^{-}}\left(\frac{a_{2}}{d_{1}}\left(\zeta_{2}^{-}\right)^{\alpha_{2}}+\zeta_{1}^{+}\right)$, which implies the desired result.

Subcase 3. $\frac{\tilde{d}_{\varepsilon}\left(x_{\varepsilon}, y_{\varepsilon}\right)}{\varepsilon} \geq \frac{\zeta_{2}^{-}}{K_{2}}$ : the desired inequality comes from the one proven in Subcase 2 and the fact that $k_{1} d_{1} \geq K_{2} b_{2}$.

\section{References}

[1] Achdou, Y., Camilli, F., Cutrì, A., Tchou, N.: Hamilton-Jacobi equations on networks. In: Proceedings of the 18th IFAC World Congress, Milano, vol. 18, part 1 (2011) 
[2] Bardi, M., Capuzzo-Dolcetta, I.: Optimal control and viscosity solutions of Hamilton-Jacobi-Bellman equations. In: Systems and Control: Foundations \& Applications, Birkhäuser, Boston, MA (1997) (with appendices by Maurizio Falcone and Pierpaolo Soravia)

[3] Bressan, A., Hong, Y.: Optimal control problems on stratified domains. Netw. Heterog. Media 2(2), 313-331 (electronic) (2007)

[4] Capuzzo-Dolcetta, I., Lions, P.-L.: Hamilton-Jacobi equations with state constraints. Trans. Am. Math. Soc. 318(2), 643-683 (1990)

[5] Deckelnick, K., Elliott, C.M.: Uniqueness and error analysis for Hamilton-Jacobi equations with discontinuities. Interfaces Free Bound. 6(3), 329-349 (2004)

[6] Engel, K-J., Kramar, F.M., Nagel, R., Sikolya, E.: Vertex control of flows in networks. Netw. Heterog. Media 3(4), 709-722 (2008)

[7] Frankowska, H., Plaskacz, S.: Hamilton-Jacobi equations for infinite horizon control problems with state constraints. In: Calculus of Variations and Optimal Control. Chapman \& Hall/CRC Res, Haifa (1998). Notes Math., vol. 411, Chapman \& Hall/CRC, Boca Raton, FL, pp. 97-116 (2000)

[8] Frankowska, H., Plaskacz, S.: Semicontinuous solutions of Hamilton-JacobiBellman equations with degenerate state constraints. J. Math. Anal. Appl. 251(2), 818-838 (2000)

[9] Garavello, M., Piccoli, B.: Traffic flow on networks. In: AIMS Series on Applied Mathematics, vol. 1, American Institute of Mathematical Sciences (AIMS), Springfield, MO, Conservation laws models (2006)

[10] Imbert, C., Monneau, R., Zidani, H.: Hamilton-Jacobi approach to junction problems and application to traffic flows. preprint hal 00569010

[11] Ishii, H., Koike, S.: A new formulation of state constraint problems for first-order PDEs. SIAM J. Control Optim. 34(2), 554-571 (1996)

[12] Koike, S.: On the Bellman equations with varying control. Bull. Aust. Math. Soc. 53(1), 51-62 (1996)

[13] Lions, P.-L.: Generalized solutions of Hamilton-Jacobi equations. Pitman (Advanced Publishing Program), Boston, MA (1982)

[14] Nicaise, S.: Elliptic operators on elementary ramified spaces. Integral Equ. Oper. Theory 11(2), 230-257 (1988)

[15] Pokornyi, Yu.V., Borovskikh, A.V.: Differential equations on networks (geometric graphs). J. Math. Sci. NY. 119(6), 691-718 Differential equations on networks (2004)

[16] Schieborn, D.: Viscosity solutions of Hamilton-Jacobi equations of Eikonal type on ramified spaces. PhD thesis, University of Tübingen (2006) 
[17] Soner, H.M.: Optimal control with state-space constraint. I. SIAM J Control Optim. 24(3), 552-561 (1986)

[18] Soner, H.M.: Optimal control with state-space constraint. II. SIAM J Control Optim. 24(6), 1110-1122 (1986)

[19] Soravia, P.: Degenerate eikonal equations with discontinuous refraction index. ESAIM Control Optim. Calc. Var. 12(2), 216-230 (electronic) (2006)

[20] Tourin, A.: A comparison theorem for a piecewise Lipschitz continuous Hamiltonian and application to shape-from-shading problems. Numer. Math. 62(1), 75$85(1992)$

Yves Achdou

Laboratoire Jacques-Louis Lions

UMR 7598

UPMC

CNRS

Université Paris Diderot

Sorbonne Paris Cité

75205 Paris

France

e-mail: achdou@ljll.univ-paris-diderot.fr

Fabio Camilli

Dipartimento di Metodi e Modelli Matematici per le Scienze Applicate

Università Roma "La Sapienza"

Via Antonio Scarpa 16

00161 Rome

Italy

e-mail: camilli@dmmm.uniroma1.it

Alessandra Cutrì

Dipartimento di Matematica

Università 'Tor Vergata' di Roma

00133 Rome

Italy

e-mail: cutri@mat.uniroma2.it

Nicoletta Tchou

IRMAR, Université de Rennes 1

Rennes

France

e-mail: nicoletta.tchou@univ-rennes1.fr

Received: 11 July 2011.

Accepted: 20 February 2012. 